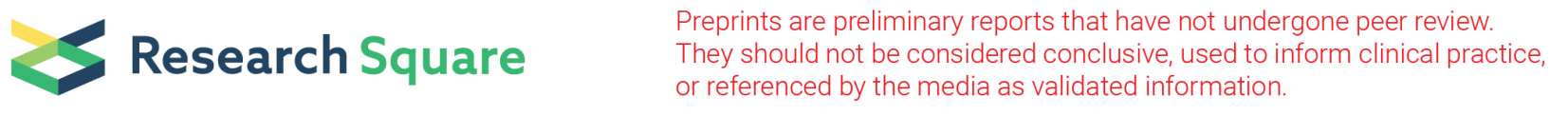

\title{
The rhizosphere microbiota of the zinc and cadmium hyperaccumulators Arabidopsis halleri and Noccaea caerulescens is highly convergent in Prayon (Belgium)
}

\author{
Amandine R. Bertrand \\ Université de Liège: Universite de Liege \\ Emilie Detry \\ Université de Liège: Universite de Liege \\ Cécile Nouet \\ Université de Liège: Universite de Liege \\ Marc Hanikenne \\ Université de Liège: Universite de Liege \\ Denis BAURAIN ( $\sim$ denis.baurain@uliege.be) \\ University of Liège https://orcid.org/0000-0003-2388-6185
}

Research article

Keywords: metabarcoding, soil, metallophyte, elemental profiling, gene expression, metal

Posted Date: October 19th, 2021

DOI: https://doi.org/10.21203/rs.3.rs-968648/v1

License: (c) (1) This work is licensed under a Creative Commons Attribution 4.0 International License. Read Full License 


\section{Abstract}

The Prayon site is known as a zinc-polluted area where two zinc and cadmium hyperaccumulator plant species currently coexist, although Arabidopsis halleri was introduced more recently than Noccaea caerulescens. While soil microorganisms may influence metal uptake, the microbial community present in the rhizosphere of hyperaccumulators remains poorly known. Plants of both species were sampled with their bulk and rhizosphere soil from different plots of the Prayon site. Soil components (ionome, pH, water composition, temperature) were analyzed, as well as shoot ionome and expression levels of metal transporter genes ( HMA3 , HMA4 , ZIP4 / ZNT1 , ZIP6, MTP1). The taxonomic diversity of the microorganisms in soil samples was then determined by 16S rRNA metabarcoding and compared at the Operational Taxonomy Unit (OTU) level and across different taxonomic levels. Our elemental analyses confirmed that the site is still highly contaminated with zinc and cadmium and that both plant species indeed hyperaccumulate these elements in situ . Although the pollution is overall high, it is heterogenous at the site scale and correlates with the expression of some metal transporter genes. Metabarcoding analyses revealed a decreasing gradient of microbial diversity, with more OTUs discovered in the rhizosphere than in the soil bulk, especially at the bottom of the cores. However, the variability gradient increases with the distance from roots. Using an ad hoc pseudo-taxonomy to bypass the biases caused by a high proportion of unclassified and unknown OTUs, we identified Chloroflexi, Armatimonadetes, Pirellulaceae, Gemmatimonadetes and Chitinophagaceae as the drivers of the differences in the gradient along the cores. In contrast, no significant difference was identified between the rhizosphere composition of $\mathrm{A}$. halleri and $\mathrm{N}$. caerulescens. This suggests that, despite their distinct colonization history in Prayon, the two plant species have now recruited highly convergent microbial communities in the rhizosphere.

\section{Introduction}

Angiosperms, i.e., flowering plants, are broadly distributed in a large variety of environments. One of such niches is metal-polluted soils, i.e., soils with an elevated concentration of metals, often of geogenic (e.g., for serpentine, Ni-rich, outcrops) or anthropogenic origin. In the latter case, these sites have been polluted either by mining activities, or by metal-transforming factories or through atmospheric deposits resulting from these activities. Plants living on metalpolluted sites are hypertolerant (Antonovics et al., 1971; Ernst, 2006): they can survive at metal concentrations much higher than the majority of plants.

Among those hypertolerant plants, some are also hyperaccumulators: they have the unique ability to take up metals from the ground and accumulate them within their leaves at high concentration (e.g., $>0.3 \%$ Zn or $0.01 \%$ Cd in shoot dry weight, SDW) (Hanikenne and Nouet, 2011; Krämer, 2010; Merlot et al., 2018). Such extremophile organisms are interesting for phytoremediation but also phytomining or biofortification (Clemens, 2017; Clemens et al., 2002; Lopez et al., 2019; Salt et al., 1998), i.e., the use of plants to clean up metal-contaminated sites, to mine metals or to enrich food for populations with element deficiencies, respectively. Two extensively studied hyperaccumulators are Arabidopsis halleri (L.) O'Kane \& Al-Shesbaz and Noccaea caerulescens (J.Presl \& C.Presl) F.K.Mey., 1973. These two Brassicaceae are plant models for zinc and cadmium hyperaccumulation and are able to live on polluted and unpolluted soils. However their adaptation to metal hyperaccumulation originates from independent evolutionary events (Corso et al., 2018; Gonneau et al., 2017; Merlot et al., 2018; Pauwels et al., 2012; Schvartzman et al., 2018).

More and more molecular actors underlying metal hyperaccumulation have been identified over the last 15 years (Hanikenne and Nouet, 2011; Krämer, 2010; Merlot et al., 2018). Even though studies have shown the importance of root-associated microorganisms in this process (Li et al., 2007; Ma et al., 2011; Sessitsch et al., 2013), little is known about the rhizosphere of metal hyperaccumulators. This could be explained by the complexity of this micro-ecosystem (Alford et al., 2010). Yet, microorganisms are known to play a role in the physiology of diverse plants (such as rice, Pseudostellaria heterophylla or Amaranthus albus (Fitzpatrick et al., 2018; Long and Yao, 2019; Wu et al., 2019; Yu et al., 2019)), including a role in processes related to metal tolerance (Ullah et al., 2015).

Albeit A. halleri does not rely on mycorrhizal symbiosis (Delaux et al., 2014; Wang and Qiu, 2006), it can associate with soil bacteria in zinc/cadmium contaminated areas, and the associated community depends on the level of metal contamination of the soil (Gomez-Balderas et al., 2014, p.). Moreover, rhizosphere bacteria may influence metal uptake by A. halleri (Farinati et al., 2011; Muehe et al., 2015). However, the taxonomic composition (Borymski et al., 2018) of rhizosphere microbial communities remains poorly known.

Our knowledge of the rhizosphere microbiota of $N$. caerulescens is even more limited, as available studies are more focused on the physical and chemical soil components in the rhizosphere than on the microbial communities themselves (Luo et al., 2018; Rosenfeld et al., 2018; Yang et al., 2011). Nevertheless, one study conducted in an agricultural soil prepared at this effect suggested that rhizosphere bacteria may also influence metal uptake by $N$. caerulescens (Whiting et al., 2001).

As some rhizosphere microorganisms are species-specific (Borymski et al., 2018; Fitzpatrick et al., 2018), it raises the question of whether the convergent evolution of hyperaccumulation in A. halleri and N. caerulescens extends to the microbial populations colonizing their rhizosphere. Yet, this is challenging to test because their geographic distribution does not overlap: $N$. caerulescens lives in the northwestern and western parts of Europe while $A$. halleri is found in more central and eastern countries of the continent (Krämer, 2010).

However, the two hyperaccumulators in fact coexist in one site in Belgium. This location is a small area of a vast zinc-polluted site in Prayon (near Liège, Fig. S1a). N. caerulescens massively colonizes the site, whereas $A$. halleri has a more limited distribution (Fig. S1c). To our knowledge, there has been no soil or flore characterization done in Prayon since 2001 (Meerts and Grommesch, 2001) and very few studies had been carried out previously (Denaeyer-De Smet and Duvigneaud, 1974; Duvigneaud and Jortay, 1987; Ramaut, 1964).

Historically, metal-oriented human activities in Prayon date back to 1503 . However, the industrial activity related to zinc only began in 1829 and led to a rapid transformation of the vegetation. The activity progressively increased between 1883 and 1913, a period during which trees disappeared and metallophytes appeared (Duvigneaud and Jortay, 1987). Atmospheric and soil pollution caused by the zinc-ore smelter were first described in 1964 (Ramaut, 1964 ). In that 
study, the zinc concentration (Fig. S1b, red dot) was measured at $540 \mathrm{mg} \mathrm{kg}^{-1}$ of dry soil (DW), i.e., more than twice the concentration considered "normal" at the time.

Consequences on the vegetation were already visible: the destruction of the original wooded flora, i.e., found before the zinc-ore smelter activity, was obvious, and hypertolerant plants were beginning to colonize the area with, at some places, $N$. caerulescens being the only plant species found (Ramaut, 1964). In 1974, the hyperaccumulator was largely present over the site. The soil pH (Fig. S1b, red dot) was between 5.5 and 6.6 and zinc concentrations had increased to values between 10,000 and $12,000 \mathrm{mg} \mathrm{kg}^{-1}$ (DW). Zinc concentration in N. caerulescens leaves was 23,950 mg kg-1 (DW) (Denaeyer-De Smet and Duvigneaud, 1974).

When the factory shut down in 1970, some of the original herbaceous species (e.g., Mercurialis perennis) eventually managed to recolonize the site but not all of them. At that time, N. caerulescens was well implanted on the polluted soil around the zinc-ore smelter. Some individuals were collected in 1987 in order to determine zinc, cadmium and lead leaf concentrations, which were measured at 24,700, 1,465 and $800 \mathrm{mg} \mathrm{kg}^{-1}$, respectively (Duvigneaud and Jortay, 1987). However, as pollution was quite widespread (hundreds of hectares, up to 2 km downwind, Fig. S1a) (Ramaut, 1964), we do not know precisely from which area of the Prayon site those individuals were sampled, even if the zinc concentration was similar to those obtained in 1974 (Denaeyer-De Smet and Duvigneaud, 1974) in the area of interest (Fig. S1b).

In 2001, the latest study in Prayon showed that there was a large variation of soil parameters across the whole site. Measurements performed on calcareous samples (Fig. S1a) varied from 5.02 to 6.18 for $\mathrm{pH}$ and from 2100 to $7800 \mathrm{mg}$ of extractable zinc $\mathrm{kg}^{-1}$ (DW). Moreover, based on seed bank composition and abundance, the study suggested that large changes were not to be expected in the floristic composition in Prayon in the following years, and that indeed seems to have been the case until now, 20 year later (Meerts and Grommesch, 2001).

Concerning A. halleri on this site (Fig. S1c), the first observation dates back to the early 2000's, according to naturalist accounts. Ironically, it might have been accidentally introduced by botanists scouting the site for $N$. caerulescens, but this hypothesis has not been supported by hard evidence so far.

Since A. halleri and N. caerulescens now coexist in Prayon, we sampled plants of both species at different plots with their bulk and rhizosphere soil and analyzed shoot and soil components as well as the taxonomic diversity present in soil samples. This revealed significant differences in microbiota between samples found at increasing distances of the roots, whereas the rhizospheres of the two plant species appeared to have a convergent microbial community.

\section{Material And Methods}

\section{Field sampling, sample preparation and characterization}

Samples from the rhizosphere of both A. halleri and N. caerulescens were collected on the 6 of June 2018 at the same metallicolous site in Belgium (Prayon, near Liège), after more than two weeks of sunny weather (around $20^{\circ} \mathrm{C}$ ). The field sampling strategy (Fig. 1) consisted in choosing 6 random sampling plots within the zone where $A$. halleri was found, i.e., at the bottom of the slope, close to a fishery pond (Fig. S1). Each plot had a surface of $1 \mathrm{~m} \times 2 \mathrm{~m}$, hence $2 \mathrm{~m}{ }^{2}$. Within this perimeter, five individuals of each species, together with their soil (core of $15 \mathrm{~cm}$ deep with a diameter of $8 \mathrm{~cm}$ ) were collected and divided into four parts: shoot, rhizosphere, bulk 1 and bulk 2 (respectively, the upper and bottom parts of the core), which were each pooled per species and plot. Shoot samples for gene expression analysis were immediately crushed and stored in RNAlater ${ }^{\circledR}$ Invitrogen (ThermoFisher). GPS coordinates and soil temperature were recorded at harvest (resulting from the mean of five measures carried out at each corner and center of each plot) (Table S1).

Shoot samples were submitted to element analysis by Inductively-Coupled Plasma Atomic Emission Spectrometry (ICP-AES), as described in (Becher et al., 2004; Nouet et al., 2015; Talke, 2006). Total RNAs were also extracted from shoot tissues using the NucleoSpin® RNA Plant Kit (Macherey-Nagel). qPCR analyses were carried out as previously described (Nouet et al., 2015). Briefly, cDNAs were synthesized with the RevertAid H Minus First Strand cDNA Synthesis Kit (ThermoFisher Scientific) using Oligo(dT) and $1 \mu \mathrm{g}$ of total RNAs. 50-fold diluted cDNA were used to perform the PCR reactions in triplicate for each sample/primer combinations with a QuantStudio ${ }^{\text {TM }} 5$ Real-Time PCR System (Applied Biosystems) with the Low ROX SYBR ${ }^{8}$ MasterMix dTTP blue mix $\left(\right.$ Takyon $^{T M}$ ). Quality of PCR amplification was visually controlled (melting and amplification curves) using the QuantStudio ${ }^{T M}$ Design and Analysis Software v1.4.2. Relative transcript level normalization was performed with the $2^{-\Delta \Delta C t}$ method with the qBase software (Hellemans et al., 2007) using AT1G58050 and UBQ13 (A. halleri (Spielmann et al., 2020)) and Actin1 and Clathrin (N. caerulescens (Gendre et al., 2006; Schneider et al., 2013)) as reference genes, respectively (Table $\mathbf{S} 2)$.

Rhizosphere soil samples were isolated by adapting the method of (Edwards et al., 2015). Briefly, roots were placed within a sterile Phosphate Buffer Saline (PBS) solution ( $\left.\mathrm{NaCl} 137 \mathrm{mM}, \mathrm{KCl} 2.7 \mathrm{mM}, \mathrm{Na}_{2} \mathrm{HPO}_{4} 10 \mathrm{mM}, \mathrm{KH}_{2} \mathrm{PO}_{4} 1.76 \mathrm{mM}, \mathrm{pH} 7.4\right)$ then the solution was filtered through a $40-\mu \mathrm{m}$ Nylon filter and centrifuged at $5000 \mathrm{x}$ g during 10 minutes. Finally, the supernatant was removed before storage at $-80^{\circ} \mathrm{C}$.

Bulks were first sieved using a mesh of $2 \mathrm{~mm}$. For each bulk sample, a small part was stored at $-80^{\circ} \mathrm{C}$ (in order to extract DNA) while the major part was weighed (fresh weight without stone $=\mathrm{FW}$ ) before drying out for several weeks at $60^{\circ} \mathrm{C}$. Dry soil was used to measure $\mathrm{pH}$, water content, as well as exchangeable and extractable elements (Hendershot and Duquette, 1986; Stein et al., 2017). They were then weighed again (dry weight = DW).

The percentage of water content in bulks was computed as (FW-DW)/FW*100 while pH was determined following (Stein et al., 2017). Briefly, $3 \mathrm{~g}$ of dry soil was mixed with $7.5 \mathrm{~mL}$ of $\mathrm{CaCl}_{2}(0.01 \mathrm{M})$ and shaked over the weekend. Samples were then centrifuged at $2000 \mathrm{xg}$ during 2 min. $\mathrm{pH}$ was measured in the supernatant (with inoLab pH 7110). The procedure was repeated five times for each sample and means were computed. The extraction of mineral elements from the soil was carried out with $\mathrm{BaCl}_{2} 0.1 \mathrm{M}$ (exchangeable elements) and with $\mathrm{HCl} 0.1 \mathrm{M}$ (extractable elements), as detailed in (Hendershot and Duquette, 
1986; Stein et al., 2017). Briefly, $1 \mathrm{~g}$ of dry soil was shaken for $2 \mathrm{~h}$ and $30 \mathrm{~min}$, respectively, then centrifuged and filtered through Whatman VWR 42 filter paper. Finally, samples were submitted to element analysis by ICP-AES as described in (Nouet et al., 2015).

All soil samples were used for DNA extraction using the Qiagen DNeasy PowerSoil Pro Kit with the QIAcube robot and the IRT protocol. One extraction blank was generated for each plot. Small subunit ribosomal RNA genes (SSU rRNA 16S/18S) were amplified with a universal primer pair (515f modified / 926r (Walters et al., 2016): TCGTCGGCAGCGTCAGATGTGTATAAGAGACAGGTGYCAGCMGCCGCGGTAA /

GTCTCGTGGGCTCGGAGATGTGTATAAGAGACAGCCGYCAATTYMTTTRAGTTT). Amplifications with bacterial-specific (S-D-Bact-0517-a-S-17 / S-D-Bact-1061a-A-17 (Maciejewska et al., 2018): TCGTCGGCAGCGTCAGATGTGTATAAGAGACAGGCCAGCAGCCGCGGTAA /

GTCTCGTGGGCTCGGAGATGTGTATAAGAGACAGCRRCACGAGCTGACGAC) and archaeal-specific (Uni519F / Arc908r (Jørgensen et al., 2013): TCGTCGGCAGCGTCAGATGTGTATAAGAGACAGCAGCMGCCGCGGTAA / GTCTCGTGGGCTCGGAGATGTGTATAAGAGACAGCCCGCCAATTCCTTTAAGTT) primer pairs were also performed in an attempt to corroborate the results. At least one PCR blank was included with each primer pair. Universal and archaeal primers were designed to amplify V4-V5 regions while bacterial primers targeted V4-V6 regions. The program used for PCR amplification is provided in Table S3.

The amplicons were then sequenced (paired-end, 2 × 300 bp) using the Illumina MiSeq 600-cycle V3. Library preparation was done following the $16 \mathrm{~S}$ metagenomic workflow from Illumina (IlluminaTechnicalSupport, n.d.). Raw reads were deposited in the SRA database with BioproJect number PRJNA717797.

\section{Bioinformatic preprocessing}

The global pipeline is described in Fig. S2 and our custom scripts are available in the figshare project (Bertrand et al., 2021) at [https://doi.org/10.6084/m9.figshare.14371781.v1].

Briefly, reads were merged, stripped from primer sequences, oriented, quality filtered then pooled together using the usearch (Edgar, 2010)

(v11.0.667_i86linux64) software suite, following the pipeline described in the documentation (Fig. S2). Clustering into operational taxonomic units (OTUs) was carried out with the UPARSE algorithm of usearch (Edgar, 2013). The same software suite was used to create an OTU table and its normalization by random read sub-sampling (allowing us to analyze the same total number of reads per sample).

A filtration of OTUs was also done with the script prune-outliers.pl, now distributed with the software Forty-Two (D. Baurain; https://metacpan.org/dist/BioMUST-Apps-FortyTwo). This was an additional quality control to retain only OTUs that were similar to at least one of the other OTUs, i.e., it kept SSU rRNA sequences and discarded non-homologous sequences potentially produced by the usearch pipeline. It is based on a mutual detection by all-vs-all BLASTN searches across all samples, given that SSU rRNA sequences are conserved enough at the nucleotide level to match those of another organism, even if quite far from a taxonomic point of view.

Taxonomic affiliation was carried out with sina (Pruesse, 2012) against the SILVA (Pruesse et al., 2007) Ref NR 99 (release 132) database. Taxonomic remapping of SILVA lineages to NCBI Taxonomy for further analyses was performed using fetch-tax.pl from Bio::MUST::Core utilities; D. Baurain; https://metacpan.org/dist/Bio-MUST-Core).

Moreover, a phylogenetic tree of the filtered OTUs was computed with RAxML (Stamatakis, 2014) v8.1.17 after multiple sequence alignment using mafft v7.453 (Katoh, 2002; Katoh and Standley, 2013). In order to emulate a taxonomy with nested levels for all OTUs, including unknown and poorly known organisms, patristic distances were extracted from the tree and fed to the R package MCL (Jäger, 2015) to partition OTUs into six sets of clusters of varying number and sizes. Those different sets were obtained by fixing the inflation parameter at 2.5 and by giving the "phylo" threshold the values $0.004,0.008$, $0.010,0.015,0.020,0.025$. In the following, the resulting nested levels will be referred to as pseudo-taxonomy.

As shown in Fig. S2, a pilot analysis was first carried out to determine the optimal sequencing depth. To this end, a second step of filtration allowed us to keep only OTUs with eight or more supporting reads, so as to filter out chimeras (Fig. S2). Rarefaction curves were then generated with the R package ggplot2 (Wickham, 2009). Barplots showing the results from the clustering analyses were also generated with the R package ggplot2 (Wickham, 2009 ).

\section{Diversity and abundance analyses}

\section{Taxonomic analysis}

Alpha and beta diversity were computed with a custom Perl script: compute-stat-diversity.pl based on Bio::MUST::Core and Bio::Community modules (F. Angly; https://metacpan.org/dist/Bio-Community). Those diversity measures were then analyzed graphically using the R package ggplot2 (Wickham, 2009 ) (graphstat-alpha-div-tax.R and graph-stat-beta-div-tax.R) with ANOVA tests. Taxonomic diversity across samples was also studied through NMDS using the R package vegan (Oksanen et al., 2018) with a discretization in 10 categories following the OTU abundances (nmds_diversity_univ30k.R). The R package ggplot2 (Wickham, 2009) was used for barplots (barplot_all_tax.R) and paired $t$-tests were computed. Venn diagrams were computed using the R package eulerr (Larsson, 2019). All scripts are available at [https://doi.org/10.6084/m9.figshare.14376773.v1].

A database was built with sqlite3 to efficiently store and analyze all the data gathered in the experiments reported here. This database was called MetaRhizoMet.sdb (Fig. S3 and Suppl. File at [https://doi.org/10.6084/m9.figshare.14258528.v1]) and is used by the different scripts previously mentioned. 


\section{Correlation with soil and plant metadata}

Variability within and between sample metadata was analyzed using the script fig_shoot_soil_param.R based on vegan (Oksanen et al., 2018) and ggplot2 (Wickham, 2009) packages. ANOVA tests were computed. Correlations were computed with the vegan (Oksanen et al., 2018) package.

\section{Results And Discussion Characterization of the Prayon site}

The two hyperaccumulators A. halleri and N. caerulescens colonize the Prayon site (near Liège, Belgium, Fig. S1), enabling the comparison of their hyperaccumulation capacities in a natural location and the study of the specificities of the microbial composition of their rhizospheres. Shoot, rhizosphere, bulk 1 and bulk 2 (top and bottom of the core) samples of each plant species were collected in six plots on this calamine site (Fig. 1) in order to determine if their convergent evolution was reflected in the rhizosphere microbiota.

\section{Soil}

$\mathrm{pH}$, water content, temperature and element composition, including zinc and cadmium, were measured in bulk 1 and bulk 2 soil samples (Fig. 2 ). pH and temperature were stable through the six plots: temperature ranged between 17.8 and $19.2^{\circ} \mathrm{C}$ and pH between 6.04 and 6.59 . This pH is lower than in other studies of $A$. halleri (Corso et al., 2018; Stein et al., 2017) and some of $N$. caerulescens (Luo et al., 2018; Rees et al., 2019; Rosenfeld et al., 2018) even if it seems more common for N. caerulescens (Luo et al., 2018; Rosenfeld et al., 2018). Water content was slightly more variable, ranging between 29.52 and $40.47 \%$ of fresh weight for bulk 1 and between 27.41 and $58.14 \%$ for bulk 2, which is coherent with previous study (Luo et al., 2018).

Overall, a limited variation is observed for extractable $(\mathrm{HCl})$ and exchangeable $\left(\mathrm{BaCl}_{2}\right)$ element concentrations between soil cores associated with $A$. halleri or $N$. caerulescens. Between bulk 1 and bulk 2 samples, only a few extractable elements varied (Al, Fe and $\mathrm{Na}$ ), in contrast to exchangeable elements. Fe and $\mathrm{Na}$ are the most variable elements across plots (Fig. S4). As expected based on the history of the site (Denaeyer-De Smet and Duvigneaud, 1974; Duvigneaud and Jortay, 1987; Ramaut, 1964), a large zinc pollution is measured. Current values are largely higher than those measured in $1974^{33}$, even with respect to exchangeable rather than extractable zinc. This could be explained by the differences in the techniques used since the activity of the factory stopped in 1970.

Moreover, extractable Zn concentrations (i.e., 4,934 to $25,051 \mathrm{mg} \mathrm{kg}^{-1}$ for bulk 1 and 6,326 to $13,829 \mathrm{mg} \mathrm{kg}^{-1}$ for bulk 2) are largely superior to the reference values for Wallonia soil ("Législation/décret gestion et assainissement sols," n.d.). Indeed, natural soil is expected to contain a maximum Zn concentration of $196 \mathrm{mg} \mathrm{kg}^{-1}$ (DW) and the threshold for necessary intervention on industrial soil set by Wallonia regulations is $3,000 \mathrm{mg} \mathrm{kg}^{-1}$. The same is true for cadmium, for which the intervention threshold is fixed at $20 \mathrm{mg} \mathrm{kg}^{-1}$. Here in Prayon, even the exchangeable concentrations $\left(42.45-174.77 \mathrm{mg} \mathrm{kg}^{-1}\right)$ are largely above this limitation. This is not the case for $\mathrm{Pb}$ and $\mathrm{Cu}$, which have high values (extractable concentrations are respectively 500-1500 and 250-625 mg kg-1) but remain below intervention thresholds (respectively 1840 and $600 \mathrm{mg} \mathrm{kg}^{-1}$ ).

If we compare with concentrations in natural soils reported in other studies, $\mathrm{Zn}, \mathrm{Cu}$ and $\mathrm{Cd}$ exchangeable concentrations are in the higher range of contaminated sites in which $A$. halleri can be found, and Pb exchangeable concentration is also within metallicolous site ranges (Stein et al., 2017). Moreover, $\mathrm{Zn}$ and $\mathrm{Cd}$ extractable concentrations are above most natural metallicolous sites colonized by $A$. halleri. Concerning $N$. caerulescens, the Prayon values of exchangeable $\mathrm{Cd}$ and $\mathrm{Zn}$ surpass total soil concentration found in other studies of natural soil (Rees et al., 2019) except one that is in the same range (Rosenfeld et al., 2018). This could explain the low plant diversity found in the sampling site. Along with $A$. halleri and $N$. caerulescens, only Armeria maritima, Viola lutea and Festuca ovina were identified, three metal hypertolerant but not hyperaccumulator plants.

\section{Hyperaccumulation in plant shoot}

Soil parameters influence the accumulation levels in shoot, thus shoot samples (composed of five pooled individuals) were collected within six 2 - $\mathrm{m}^{2}$ plots for each species (Fig. 1) and submitted to ionome profiling and quantification of metal transporter gene expression.

Micro- and macronutrient shoot concentrations were mostly similar between the two plant species, with the exception of Mg, P and Mn concentrations, which were higher in A. halleri than in N. caerulescens (Fig. 3). Metal accumulation in shoot differed significantly between the two species only for Cd, where $N$. caerulescens hyperaccumulated more. In contrast, $\mathrm{Zn}$ and $\mathrm{Pb}$ concentration ranges did overlap between shoots of both plants. Finally, $A$. halleri and $N$. caerulescens hyperaccumulated both $\mathrm{Zn}$ and Cd above the defined hyperaccumulation thresholds of 3,000 and $100 \mathrm{mg} \mathrm{kg}^{-1}$ (DW), respectively (Krämer, 2010). Even if $N$. caerulescens is known to hyperaccumulate $\mathrm{Cd}$, it is not the case for all ecotypes. It is particularly the case of the Prayon ecotype, which was described as unable to hyperaccumulate $\mathrm{Cd}$ (Rosenfeld et al., 2018). However, most studies used soils with lower Cd concentration, therefore limiting $\mathrm{Cd}$ accumulation. Furthermore, shoot Cd accumulation in the Prayon site is much lower than observed in the Ganges ecotype of N. caerulescens, a bona-fide Cd hyperaccumulator ecotype (Halimaa et al., 2019).

We next examined the expression levels of metal transporter genes that were previously shown to be highly expressed in the shoot of $A$. halleri and $N$. caerulescens and linked to hyperaccumulation and/or tolerance (Hanikenne et al., 2008; Talke, 2006; van de Mortel et al., 2006): HMA3 (Zn and Cd vacuolar storage (Talke, 2006; Ueno et al., 2011)), HMA4 (Zn and Cd xylem (un)loading and distribution (Craciun et al., 2012; Hanikenne et al., 2008)), ZIP4/ZNT1 (Zn uptake and distribution (Lin et al., 2016; Talke, 2006)), ZIP6 (Cd tolerance (Spielmann et al., 2020; Wu et al., 2009)) and MTP1 (Zn vacuolar storage (Dräger et al., 2004; Gustin et al., 2009; Shahzad et al., 2010)). Note that information on the expression of those genes in plants in the field is very limited. The HMA3, 
HMA4 and ZIP4/ZNT1 genes showed the largest variation in expression level (up to 3.4-fold) throughout samples, both in A. halleri and N. caerulescens. MTP1 seemed also to vary but to a lesser extent (Fig. S5, S6). Correlations of gene expression were computed with the element concentrations in shoot tissues. In A. halleri, a relation between the Pb concentration and ZIP4 expression is observed ( $p$-value=0.0069), as well as between $\mathrm{P}$ and MTP1 ( $p$ value $=0.047)$. In $N$. caerulescens, a correlation is observed between the Mn concentration and the HMA4 expression ( $p$-value $=0.00046)$ and between $\mathrm{Mg}$, $\mathrm{Pb}$ and $\mathrm{K}$ and MTP1 ( $p$-value=0.02, 0.035 and 0.062, respectively), as well as Pb and HMA4 ( $p$-value=0.052) (Fig. S7).

Correlations were also observed between the soil concentration of some elements and the expression of related transporter genes. In $A$. halleri, it is the case between the concentration of $\mathrm{Zn}$ and the expression of ZIP4 ( $p$-value=0.0099) as well as between $\mathrm{Cd}$ and HMA4, Cd and ZIP6, Na and ZIP4 ( $p$-value=0.019, 0.031 and 0.049 , respectively). In N. caerulescens, correlations were found between ZNT1, HMA3, ZIP6 and HMA4 and several elements (Fig. S8).

Those correlations are relatively weak and at this point, disentangling causal relationships between gene expression and metal concentrations would be hazardous. Differences in the observations in A. halleri and N. caerulescens might be explained by their different physiology (Merlot et al., 2018), including distinct metal storage sites, in mesophyll and in epidermis for $A$. halleri and N. caerulescens, respectively.

Interestingly, in spite of a generally high level of zinc and cadmium pollution, the heterogeneity of the site of Prayon seems to correlate with the expression of some metal transporter genes. However, no correlation was found between the amount of zinc present in shoot and the amount present in soil, in contrast to Stein et al. 2017 (Stein et al., 2017) (Fig. S9).

\section{Microbial diversity}

\section{On the optimal sequencing depth}

Since microorganisms present in the rhizosphere impact plant physiology and can be species-specific, a major goal of this study was to characterize as accurately as possible the microbial diversity of our soil samples, i.e., the number and relative abundance of each OTU or taxon. To this end, DNA was extracted from all soil samples (rhizosphere and bulks) and SSU rRNA (16S/18S) gene regions were amplified either with universal primers or with bacterial and archaeal-specific primer pairs for corroboration. Amplicons were sequenced using the Illumina MiSeq technology.

We first tested whether the planned sequencing depth enabled the identification of the whole microbial diversity in our samples. This pilot analysis was conducted on three samples (rhizosphere, bulk 1 and bulk 2) from five pooled $A$. halleri individuals sampled in the same plot. Five different depths were tested, obtained by in silico sub-sampling of the available reads, resulting in a relative depth from $1 \mathrm{x}$ to $12 \mathrm{x}$ in each of the three samples. Reads from each depth were treated independently and processed through the pipeline described in Material and methods to identify the OTUs (i.e., potential organisms) present.

As observed in Fig. S10, the number of different taxa discovered for any sequencing depth and any taxonomic level reached a different plateau. Hence, at deeper depth, more organisms are found when replaying the whole pipeline, suggesting that these rarefaction curves are not informative about our ability to discover (or not) all the microbial richness in the samples. Because we observe this phenomenon whatever the taxonomic level analyzed, it is unlikely to be (entirely) due to "new" sequences resulting from undetected chimeras or sequencing errors. Moreover, similar results were obtained with all primer pairs.

Yet, as many OTUs (up to $12.7 \%$ at the deepest depth) actually correspond to unclassified organisms according to SILVA taxonomy, it was difficult to determine whether increasing the sequencing depth really allowed sampling a significantly larger biodiversity. To address this issue, we computed a phylogenetic tree including all the discovered OTUs and labelled the leaves with the depth(s) at which each OTU was recovered (Suppl. File at [https://doi.org/10.6084/m9.figshare.14376785.v1]). This tree was then used to build a pseudo-taxonomy with five nested levels of pseudo-taxa accounting for all OTUs, including those unclassified in the SILVA taxonomy. These analyses revealed that new pseudo-taxa consistently appear at all levels when increasing depth (Fig. S11). Hence, between 16.4 and $22.7 \%$ of the clusters segmenting the phylogenetic tree are only observed at the two deepest sequencing depths.

Those results suggest that there is no good way to ascertain the optimal sequencing depth to uncover the whole microbial richness, even if some studies try different approaches to resolve this problem (Alteio et al., 2020; Fuks et al., 2018; Xiao et al., 2018). Therefore, comparison between studies or samples should take into account the depth parameter in addition to the primer pair, the sequencing method and the bioinformatics pipeline used. In the present work, the sequencing depth was eventually set at $4 x$ the depth of the pilot study, as it was the minimal depth providing a reasonable account of the real diversity.

\section{Assembly and quantification of SSU rRNA OTUs}

Altogether, a total of 36 samples (rhizosphere and bulks from both species in the six locations), along with extraction and amplification blanks, were sequenced by Illumina after amplification of a SSU rRNA fragment. The results presented in the following were obtained with the universal primer pair, which uncovered the largest richness (compared to bacterial and archaeal primer pairs). Indeed, data obtained with bacterial and archaeal primer pairs had to be normalized at 1,000 reads (instead of 30,000; see below). Moreover, they were not especially Bacteria or Archaea-specific, contrary to our expectations, and did not allow us to find additional organisms beyond those obtained with the universal primer pair. The corresponding data is nevertheless available at [https://doi.org/10.6084/m9.figshare.14258528.v1].

Reads were analyzed through the bioinformatics pipeline described in Material and methods in order to generate a normalized OTU table in which each OTU has been assigned a taxon based on SILVA taxonomy. The normalization was fixed for all samples at 30,000 reads. It was the minimal number of reads for 
which we could have a reasonable taxonomic diversity with a maximum of our samples. The resulting OTU table counts 9,796 OTUs (out of the 11,328 originally identified by usearch) and 30 out of the 36 samples were conserved. Because of the 30,000-read normalization, we have lost the bulk 2 sample from A. halleri in the second plot and five samples from the sixth plot (i.e., all except the bulk 1 from N. caerulescens). Finally, after the filtration step, 9,323 OTUs remained and were used in the following analyses.

\section{Microbial diversity at the OTU level}

The taxonomic diversity was then compared between these 30 samples. A non-metric multidimensional scaling (NMDS) was first computed to visualize whether samples had similar (or different) microbial diversity comprising the number of different organisms and their abundance (Fig. 4a). A clear separation between each part of the core is observed, i.e., between rhizosphere, bulk 1 and bulk 2 . It also shows a gradient of diversity from the roots to the bottom of the core, i.e., rhizosphere then bulk 1 (top of the core) then bulk 2 (bottom of the core). Interestingly, the projected distance between rhizosphere and bulks is larger than between the two bulks, suggesting a more different microbial diversity in the rhizosphere. Moreover, the variance within each type of sample apparently follows the same gradient.

Since the major variability, i.e., differences found in the diversity between samples, seems to be driven by the physical distance from the roots, which is in line with other studies (Praeg et al., 2019; Song et al., 2020), this analysis could hide potential differences in the microbial diversity between the rhizospheres of the two species (A. halleri and N. caerulescens). To check that possibility, another NMDS was computed for those specific samples (Fig. 4b). While samples from either plant visually form distinct groups, their separation is not very clear, suggesting only subtle differences between rhizosphere compositions, less important than the differences between sample types (i.e., rhizosphere, bulk 1 and bulk 2). This is coherent with the distribution of OTUs in Venn diagrams, according to sample type or plant species (Fig. S12).

Statistical values for alpha- and beta-diversity were then computed at the OTU level within and between samples, respectively (Fig. 5) to better understand the variations revealed in the NMDS plots. A decreasing gradient of richness is observed from rhizosphere to bulk 2 (Fig. 5a), i.e., the number of different organisms is the highest in rhizospheres, which is fully coherent with the first NMDS analysis (Fig. 4a).

However, Simpson's index of alpha-diversity, which takes into account both the number of "species" present and their abundances, highlights that the overall variability in bulk 1 is similar to the one found in the rhizosphere (Fig. S13a), even if there are more organisms that are different in the rhizosphere (Fig. 5a). Simpson's index also shows a larger variability between bulk 2 samples (Fig. S13a), which is confirmed by Euclidean distances of beta-diversity, taking abundances into account too (Fig. 5b). Similarly, beta-diversity measures also show that bulk 2 differs more from bulk 1 and rhizosphere, which are very similar in terms of Euclidean distances computed on OTUs.

In summary, the taxonomic diversity is mostly driven by the physical distance from the roots, along a decreasing gradient, rhizospheres having the highest richness and Simpson's diversity but a smaller variability between samples than bulks, with bulk 2 having the highest variability and the smallest number of different organisms. Thus, the rhizosphere appears to be a more favorable microenvironnement than bulks but with more constraints. However, those constraints appear similar between the two plant species, considering the convergence of the microbial communities observed in their rhizosphere.

\section{Microbial diversity at different taxonomic levels}

The microbial composition (top-10 taxa) was then compared between samples at different taxonomic levels (Fig. 6a). Differences appear between rhizosphere and bulks, and maybe between plant species too. At the phylum level, even if the most represented phylum is Proteobacteria for all samples, it is less abundant within bulk 2. More Bacteroidetes and Actinobacteria are present in the rhizosphere. Moreover, Acidobacteria, Chloroflexi and Firmicutes are less represented in the rhizosphere than in bulks, which may depend on metal contamination levels as suggested by observed results in the presence of severe Cdpollution in a previous study (Song et al., 2020). Interestingly, unclassified taxa (according to SILVA taxonomy) are among the 10 most represented taxa at each taxonomic level.

Based on the results obtained with OTUs (Fig. 5a), we expected to observe a larger richness in the rhizosphere. However, if we take into account the "other" taxa that are clustered together (i.e., the remaining taxa out of the 10 most abundant), it does not seem to be the case at the phylum level (Fig $6 a)$. Indeed, bulk 2 has not only more "other" phyla but also a larger proportion of unclassified and unknown (= undef_tax) organisms. This difference might be biologically genuine and explained by visualization at a higher taxonomic level (i.e., phylum) than the OTUs used previously. As a case in point, the richness shows a loss of signal and a large variability that is not in line with observations on OTUs throughout the different taxonomic levels (Fig. S14). Regarding beta-diversity measures, they are even more variable across levels (Fig. S15).

Alternatively, the systematically large proportion of unclassified and unknown organisms might also impact the analyses, considering that information loss is greater at lower taxonomic levels, i.e., it is more difficult to reliably classify an OTU at the genus level than at the class or phylum level (Table S4, Fig. 6a). In detail, 6,186 out of 9,323 identified OTUs (66\%) are classified at any level, and only 1,794 (19\%) are affiliated down to the genus level. The remaining OTUs (15\%) are classified at various higher taxonomic levels, including 350 OTUs (4\%) above the phylum level, thus remaining unknown for most practical purposes. Furthermore, taxonomic affiliation levels are heterogeneous between sample types, with more reads classified at the genus level in the rhizosphere than in bulk 2 and the opposite at the phylum level (Fig. S16, Fig. 6a).

\section{Microbial diversity with pseudo-taxonomic affiliations}

Page $7 / 18$ 
To bypass these biases created by missing taxonomic affiliations, a pseudo-taxonomy was computed following the same strategy as for the sequencing depth pilot study (see above), but this time using all OTUs remaining after filtration (9,323, Fig. S2) to compute the phylogenetic tree (Table S4). As shown in Table S5, the number of different organisms found in each pseudo-taxonomic level is constant.

With this pseudo-taxonomy, richness measures were computed anew (Fig. S17). No particular signal seems to emerge at pseudo-class and -phylum levels. However, the variability pattern observed at pseudo-species, -genus and -family levels is congruent with the pattern found with OTUs (Fig. 5a). Indeed, the richness is higher in the rhizosphere and follows a gradient of decreasing diversity through sample types. This was not observable above the OTU level when using the SILVA taxonomy (Fig. S14).

Beta-diversities were then recomputed on this pseudo-taxonomy (Fig. S18). They appear coherent both with Simpson's index (Fig. S19) and earlier analyses carried on OTUs (Fig. 5b). Indeed, there is a gradient throughout pairwise comparisons from rhizosphere to bulk 2 at the pseudo-order, -class and -phylum level, thereby confirming a smaller variability in the diversity found in the rhizosphere than in bulks, with bulk 2 being the most variable. Moreover, beta-diversity also shows a significant difference between the rhizospheres from A. halleri and $N$. caerulescens (Fig. S18) at multiple levels, as suggested by the second NMDS analysis (Fig. 4b) and the composition found with the pseudo-taxonomy (Fig. 6b). Indeed, differences (Fig. S18) between rhizosphere composition of the two plant species at pseudo-phylum, -class and -order are visually observed and some variability appears at pseudo-family and -species levels.

Euclidean distances between rhizospheres of $A$. halleri are almost systematically lower than those between rhizospheres of $N$. caerulescens (Fig. S18). This may suggest a sharper selection caused by the roots of $A$. halleri than by those of $N$. caerulescens.

\section{Differences in the microbial diversity within rhizospheres}

To investigate a possible difference of microbial composition in rhizosphere samples from both plant species, a paired $t$-test was computed across all six plots for all pseudo-taxonomic levels (Table S6a), using only the most abundant pseudo-taxa (i.e., those shown in Fig. 6b). However, not a single pseudo-taxon abundance appeared as significantly different at any taxonomic level, the best candidates being cluster 41 at the pseudo-family level and cluster 536 at the pseudo-species level, with a corrected $p$-value of 0.08 and 0.06 , respectively. Cluster 41 is composed of OTUs that were taxonomically associated to Chloroflexi (76\%) and Armatimonadetes (4\%) taxa (both from Terrabacteria group), as well as unclassified 0TUs (20\%), whereas cluster 536 is composed of OTUs assigned to Pirellulaceae (96\%) and of a few unassigned OTUs (4\%). Interestingly, those phyla are often cited when soil conditions vary (Edwards et al., 2015; Mukhtar et al., 2018; Sánchez-Marañón et al., 2017; Song et al., 2020; Sun et al., 2018; Wu et al., 2019), except Pirellulaceae, which might be an indicator of soil pH (Hermans et al., 2017).

In contrast, a comparison between bulk 1 and rhizosphere of $A$. halleri identifies 2-5 pseudo-taxa with a significant difference at each pseudo-taxonomic level (Table S6b), as does the same comparison for $N$. caerulescens samples (Table S6c). Among all those differences, Gemmatimonadetes, also varying in other studies (Sun et al., 2018; Yue et al., 2020; Zhao et al., 2020), Chloroflexi and Armatimonadetes (along with unknown and undefined organisms) are found in several clusters at various levels for $A$. halleri (cluster 435 at the pseudo-genus level, clusters 71 and 41 at pseudo-family, cluster 1 at pseudo-order and cluster 1 at pseudo-class). Cluster 536 at the pseudo-species level highlights again a difference in Pirellulaceae. Concerning the most significant differences found between bulk 1 and the rhizosphere of $N$. caerulescens, Chitinophagaceae compose the whole cluster 418 at the pseudo-species level. This phylum was previously identified as an indicator of aluminium concentration in soil (Hermans et al., 2017), but it was also shown to be mostly found in rhizosphere (Song et al., 2020).

These statistical analyses are globally in line with the results obtained at the OTU level (Fig. 4, Fig. 5) and corroborate the insights brought by our alpha- and beta-diversity analyses based on pseudo-taxonomy (Fig. S19, Fig. S18). While they demonstrate that relying on a pseudo-taxonomy can indeed be enlightening in a study like ours, they also confirm that the microbial communities of the two plant species studied in this work are essentially convergent. Such an outcome might be explained either by the spatial proximity of the roots sampled in the field and/or by the phylogenetic proximity of the corresponding plant species. Indeed, these two species belong to Brassicaceae and their rhizospheres might exert a similar selection on a possibly similar preexisting microbial community. A sampling of rhizospheres from non-hyperaccumulator plant species present on the same site could be useful to ascertain this hypothesis, such as Armeria maritima or Viola lutea. Another explanation could stand for the necessity of taking into account less abundant taxa. This would be in line with statistical differences obtained in beta-diversity (Fig. S18) and not with major taxa. Moreover, less abundant unknown and undetected taxa may be of importance in the specificity of microbiomes (Thomas and Segata, 2019).

\section{Conclusions}

The Prayon site near Liège (Belgium) is still highly contaminated with zinc and cadmium, and current values are even higher than those measured in $1974^{33}$. Because of this pollution, only five plant species are able to colonize the site. Among them are A. halleri and $N$. caerulescens, which both hyperaccumulate Zn and Cd.

Microbial diversity analyses revealed a larger number of OTUs in the rhizosphere of both A. halleri and $N$. caerulescens than in bulk soil samples (Fig. 7). The decreasing gradient of diversity, from rhizospheres to the bottom of the sampled cores, may be driven by a more favorable microenvironnement created by the roots than the highly polluted medium surrounding the roots (Hou et al., 2018; Praeg et al., 2019). Yet, this microenvironnement appears to impose selection constraints on the microbiota. This is evidenced in both plant species by a lower variability in the diversity found in the rhizosphere in comparison to the bulks, especially bulk 2 (which corresponds to the bottom of the core). 
Taxonomic analyses at the phylum level were affected by a high proportion of unclassified and unknown OTUs, which were also observed at other taxonomic levels. Those OTUs lead to biases in the information provided by the taxonomic affiliations. To bypass those biases, a pseudo-taxonomy was computed based on a global phylogenetic tree using all discovered OTUs. Consequently, alpha- and beta-diversity measures based on the pseudo-taxonomy showed a higher diversity in rhizospheres than in bulks, with bulk 2 being the most variable, as observed with the OTUs. Organisms at least partly implied in these differences belong to Chloroflexi, Armatimonadetes, Pirellulaceae, Gemmatimonadetes and Chitinophagaceae, which were previously cited as varying with soil parameters.

In contrast, no significant difference between the rhizospheres of $A$. halleri and $N$. caerulescens could be found. This indicates that the rhizosphere microbiota of the two plant species is highly convergent. Nearly significant differences may be due to organisms belonging to Chloroflexi, Armatimonadetes and Pirellulaceae, but if some compositional differences do exist, they are likely to involve unknown and unclassified organisms.

While the overall similarity of the rhizosphere of $A$. halleri and $N$. caerulescens could be explained by the proximity of the two plant species, whether in the field or at the phylogenetic level, the composition of the soil could also be responsible. Indeed, the high level of zinc and cadmium contamination on the site is likely to be the main factor influencing the microbial diversity. Therefore, it would be interesting in further studies to compare the taxonomic composition of the rhizosphere in a single hyperaccumulator plant species but throughout different soils, polluted or not.

\section{Declarations}

\section{Ethics approval and consent to participate}

Not applicable.

\section{Consent for publication}

Not applicable.

\section{Availability of data and materials}

All data generated or analysed during this study are included in this published article [and its supplementary information files].

\section{Competing interests}

The authors declare that they have no competing interests.

\section{Funding}

Funding was provided by the University of Liège grant SFRD-12/04 (DB), and F.R.S.-FNRS grants CDR J.0080.15 (DB), CDR J.0009.17 (MH), PDR T0120.18 $(\mathrm{MH})$, and CDR J.0008.20 (DB). ARB is supported by the FRIA. MH is a Senior Research Associate of F.R.S.-FNRS.

\section{Author contribution}

Conceptualization, M.H. and D.B.; methodology, A.R.B., M.H. and D.B.; software, A.R.B. and D.B.; formal analysis, A.R.B.; investigation, A.R.B., E.D. and C.N.; resources, M.H. and D.B.; data curation, A.R.B.; writing - original draft, A.R.B. and D.B.; writing - review and editing, A.R.B., C.N., M.H. and D.B.; visualization, A.R.B.; supervision, M.H. and D.B.; project administration, A.R.B.; funding acquisition, M.H. and D.B.

\section{Acknowledgements}

The authors thank Marie Schloesser and Arnaud Delgueldre for their technical assistance, Rosa Gago for her graphical assistance, the Laboratory of Plant and Microbial Ecology (ULiège) for access to ICP-AES, the Laboratoire de Génétique de la Conservation (GeCoLAB, ULiège) for access to QiaCUBE, and finally Natagora for authorizing the sampling on the Prayon site.

\section{References}

Alford, É.R., Pilon-Smits, E.A.H., Paschke, M.W., 2010. Metallophytes-a view from the rhizosphere. Plant Soil 337, 33-50. https://doi.org/10.1007/s11104-0100482-3

Alteio, L.V., Schulz, F., Seshadri, R., Varghese, N., Rodriguez-Reillo, W., Ryan, E., Goudeau, D., Eichorst, S.A., Malmstrom, R.R., Bowers, R.M., Katz, L.A., Blanchard, J.L., Woyke, T., 2020. Complementary Metagenomic Approaches Improve Reconstruction of Microbial Diversity in a Forest Soil. mSystems 5, e00768-19, /msystems/5/2/msys.00768-19.atom. https://doi.org/10.1128/mSystems.00768-19

Antonovics, J., Bradshaw, A.D., Turner, R.G., 1971. Heavy Metal Tolerance in Plants, in: Cragg, J.B. (Ed.), Advances in Ecological Research. Academic Press, pp. 1-85. https://doi.org/10.1016/S0065-2504(08)60202-0

Becher, M., Talke, I.N., Krall, L., Krämer, U., 2004. Cross-species microarray transcript profiling reveals high constitutive expression of metal homeostasis genes in shoots of the zinc hyperaccumulator Arabidopsis halleri: Transcript profiling in shoots of A. halleri. Plant J. 37, 251-268. https://doi.org/10.1046/j.1365-

313X.2003.01959.x 
Bertrand, A.R., Hanikenne, M., Baurain, D., 2021. Figshare: Bertrand et al. Microbial diversity in the rhizosphere of the zinc and cadmium hyperaccumulators Arabidopsis halleri and Noccaea caerulescens, in Prayon (Belgium) [WWW Document]. URL

https://figshare.com/projects/Bertrand_et_al_Microbial_diversity_in_the_rhizosphere_of_the_zinc_and_cadmium_hyperaccumulators_Arabidopsis_halleri_and_I

Borymski, S., Cycoń, M., Beckmann, M., Mur, L.A.J., Piotrowska-Seget, Z., 2018. Plant Species and Heavy Metals Affect Biodiversity of Microbial Communities Associated With Metal-Tolerant Plants in Metalliferous Soils. Front. Microbiol. 9. https://doi.org/10.3389/fmicb.2018.01425

Clemens, S., 2017. How metal hyperaccumulating plants can advance Zn biofortification. Plant Soil 411, 111-120. https://doi.org/10.1007/s11104-016-29203

Clemens, S., Palmgren, M.G., Krämer, U., 2002. A long way ahead: understanding and engineering plant metal accumulation. Trends Plant Sci. 7, $309-315$. https://doi.org/10.1016/S1360-1385(02)02295-1

Corso, M., Schvartzman, M.S., Guzzo, F., Souard, F., Malkowski, E., Hanikenne, M., Verbruggen, N., 2018. Contrasting cadmium resistance strategies in two metallicolous populations of Arabidopsis halleri. New Phytol. 218, 283-297. https://doi.org/10.1111/nph.14948

Craciun, A., Meyer, C.-L., Chen, J., Roosens, N., De Groodt, R., Hilson, P., Verbruggen, N., 2012. Variation in HMA4 gene copy number and expression among Noccaea caerulescens populations presenting different levels of Cd tolerance and accumulation. J. Exp. Bot. 11. https://doi.org/10.1093/jxb/ers104

Delaux, P.-M., Varala, K., Edger, P.P., Coruzzi, G.M., Pires, J.C., Ané, J.-M., 2014. Comparative Phylogenomics Uncovers the Impact of Symbiotic Associations on Host Genome Evolution. PLoS Genet. 10, e1004487. https://doi.org/10.1371/journal.pgen.1004487

Denaeyer-De Smet, S., Duvigneaud, P., 1974. Accumulation de métaux lourds toxiques dans divers écosystèmes terrestres pollués par les retombées d'origine industrielle. Bull. Société R. Bot. Belg. 107, 147-156.

Dräger, D.B., Desbrosses-Fonrouge, A.-G., Krach, C., Chardonnens, A.N., Meyer, R.C., Saumitou-Laprade, P., Krämer, U., 2004. Two genes encoding Arabidopsis halleri MTP1 metal transport proteins co-segregate with zinc tolerance and account for high MTP1 transcript levels. Plant J. 39, 425-439.

https://doi.org/10.1111/j.1365-313X.2004.02143.x

Duvigneaud, J., Jortay, A., 1987. Un site intéressant de la région liégeoise: la partie méridionale du vallon des Fonds de Forêt (Forêt et Magnée; province de Liège). Nat. Belg. 68, 33-48.

Edgar, R.C., 2013. UPARSE: highly accurate OTU sequences from microbial amplicon reads. Nat. Methods 10, 996-998. https://doi.org/10.1038/nmeth.2604

Edgar, R.C., 2010. Search and clustering orders of magnitude faster than BLAST. Bioinformatics 26, 2460-2461.

https://doi.org/10.1093/bioinformatics/btq461

Edwards, J., Johnson, C., Santos-Medellín, C., Lurie, E., Podishetty, N.K., Bhatnagar, S., Eisen, J.A., Sundaresan, V., 2015. Structure, variation, and assembly of the root-associated microbiomes of rice. Proc. Natl. Acad. Sci. 112, E911-E920. https://doi.org/10.1073/pnas.1414592112

Ernst, W.H.O., 2006. Evolution of metal tolerance in higher plants. For. Snow Landsc. Res. 80, 251-274.

Farinati, S., DalCorso, G., Panigati, M., Furini, A., 2011. Interaction between selected bacterial strains and Arabidopsis halleri modulates shoot proteome and cadmium and zinc accumulation. J. Exp. Bot. 62, 3433-3447. https://doi.org/10.1093/jxb/err015

Fitzpatrick, C.R., Copeland, J., Wang, P.W., Guttman, D.S., Kotanen, P.M., Johnson, M.T., 2018. Assembly and ecological function of the root microbiome across angiosperm plant species. Proc. Natl. Acad. Sci. 201717617.

Fuks, G., Elgart, M., Amir, A., Zeisel, A., Turnbaugh, P.J., Soen, Y., Shental, N., 2018. Combining 16S rRNA gene variable regions enables high-resolution microbial community profiling. Microbiome 6, 17. https://doi.org/10.1186/s40168-017-0396-X

Gendre, D., Czernic, P., Conéjéro, G., Pianelli, K., Briat, J.-F., Lebrun, M., Mari, S., 2006. TcYSL3, a member of the YSL gene family from the hyper-accumulator Thlaspi caerulescens, encodes a nicotianamine-Ni/Fe transporter: TcYSL3, a nicotianamine Ni/Fe transporter. Plant J. 49, 1-15.

https://doi.org/10.1111/j.1365-313X.2006.02937.x

Gomez-Balderas, C.D.C., Cochet, N., Bert, V., Tarnaud, E., Sarde, C.-O., 2014. 16S rDNA analysis of bacterial communities associated with the hyper accumulator Arabidopsis halleri grown on a Zn and Cd polluted soil. Eur. J. Soil Biol. 60, 16-23. https://doi.org/10.1016/j.ejsobi.2013.10.006

Gonneau, C., Noret, N., Godé, C., Frérot, H., Sirguey, C., Sterckeman, T., Pauwels, M., 2017. Demographic history of the trace metal hyperaccumulator Noccaea caerulescens (J. Presl and C. Presl) F. K. Mey. in Western Europe. Mol. Ecol. 26, 904-922. https://doi.org/10.1111/mec.13942

Gustin, J.L., Loureiro, M.E., Kim, D., Na, G., Tikhonova, M., Salt, D.E., 2009. MTP1-dependent Zn sequestration into shoot vacuoles suggests dual roles in Zn tolerance and accumulation in Zn-hyperaccumulating plants. Plant J. 57, 1116-1127. https://doi.org/10.1111/j.1365-313X.2008.03754.x

Halimaa, P., Blande, D., Baltzi, E., Aarts, M.G.M., Granlund, L., Keinänen, M., Kärenlampi, S.O., Kozhevnikova, A.D., Peräniemi, S., Schat, H., Seregin, I.V., Tuomainen, M., Tervahauta, A.I., 2019. Transcriptional effects of cadmium on iron homeostasis differ in calamine accessions of Noccaea caerulescens. Plant J. 97, 306-320. https://doi.org/10.1111/tpj.14121

Page $10 / 18$ 
Hanikenne, M., Nouet, C., 2011. Metal hyperaccumulation and hypertolerance: a model for plant evolutionary genomics. Curr. Opin. Plant Biol. $14,252-259$. https://doi.org/10.1016/j.pbi.2011.04.003

Hanikenne, M., Talke, I.N., Haydon, M.J., Lanz, C., Nolte, A., Motte, P., Kroymann, J., Weigel, D., Krämer, U., 2008. Evolution of metal hyperaccumulation required cis-regulatory changes and triplication of HMA4. Nature 453, 391-395. https://doi.org/10.1038/nature06877

Hellemans, J., Mortier, G., De Paepe, A., Speleman, F., Vandesompele, J., 2007. qBase relative quantification framework and software for management and automated analysis of real-time quantitative PCR data. Genome Biol. 8, R19. https://doi.org/10.1186/gb-2007-8-2-r19

Hendershot, W.H., Duquette, M., 1986. A simple barium chloride method for determining cation exchange capacity and exchangeable cations. Soil Sci. Soc. Am. J. 50, 605-608.

Hermans, S.M., Buckley, H.L., Case, B.S., Curran-Cournane, F., Taylor, M., Lear, G., 2017. Bacteria as Emerging Indicators of Soil Condition. Appl. Environ. Microbiol. 83, e02826-16, e02826-16. https://doi.org/10.1128/AEM.02826-16

Hou, D., Wang, R., Gao, X., Wang, K., Lin, Z., Ge, J., Liu, T., Wei, S., Chen, W., Xie, R., Yang, X., Lu, L., Tian, S., 2018. Cultivar-specific response of bacterial community to cadmium contamination in the rhizosphere of rice ( Oryza sativa L.). Environ. Pollut. 241, 63-73. https://doi.org/10.1016/j.envpol.2018.04.121

IlluminaTechnicalSupport, n.d. 16s-metagenomic-library-prep-guide-15044223-b.pdf [WWW Document]. URL

https://support.illumina.com/documents/documentation/chemistry_documentation/16s/16s-metagenomic-library-prep-guide-15044223-b.pdf

Jäger, M.L., 2015. MCL: Markov Cluster Algorithm.

Jørgensen, S.L., Thorseth, I.H., Pedersen, R.B., Baumberger, T., Schleper, C., 2013. Quantitative and phylogenetic study of the Deep Sea Archaeal Group in sediments of the Arctic mid-ocean spreading ridge. Front. Microbiol. 4. https://doi.org/10.3389/fmicb.2013.00299

Katoh, K., 2002. MAFFT: a novel method for rapid multiple sequence alignment based on fast Fourier transform. Nucleic Acids Res. 30, $3059-3066$. https://doi.org/10.1093/nar/gkf436

Katoh, K., Standley, D.M., 2013. MAFFT Multiple Sequence Alignment Software Version 7: Improvements in Performance and Usability. Mol. Biol. Evol. 30, 772-780. https://doi.org/10.1093/molbev/mst010

Krämer, U., 2010. Metal Hyperaccumulation in Plants. Annu. Rev. Plant Biol. 61, 517-534. https://doi.org/10.1146/annurev-arplant-042809-112156

Larsson, J., 2019. eulerr: Area-Proportional Euler and Venn Diagrams with Ellipses.

Législation/décret gestion et assainissement sols [WWW Document], n.d. URL http://environnement.wallonie.be/legis/solsoussol/sol006.htm (accessed 2.24.21).

Li, W.C., Ye, Z.H., Wong, M.H., 2007. Effects of bacteria on enhanced metal uptake of the Cd/Zn-hyperaccumulating plant, Sedum alfredii. J. Exp. Bot. 58, 4173-4182. https://doi.org/10.1093/jxb/erm274

Lin, Y.-F., Hassan, Z., Talukdar, S., Schat, H., Aarts, M.G.M., 2016. Expression of the ZNT1 Zinc Transporter from the Metal Hyperaccumulator Noccaea caerulescens Confers Enhanced Zinc and Cadmium Tolerance and Accumulation to Arabidopsis thaliana. PLOS ONE 11, e0149750.

https://doi.org/10.1371/journal.pone.0149750

Long, X.-E., Yao, H., 2019. Phosphorus Input Alters the Assembly of Rice (Oryza sativa L.) Root-Associated Communities. Microb. Ecol. https://doi.org/10.1007/s00248-019-01407-6

Lopez, S., van der Ent, A., Erskine, P.D., Echevarria, G., Morel, J.L., Lee, G., Permana, E., Benizri, E., 2019. Rhizosphere chemistry and above-ground elemental fractionation of nickel hyperaccumulator species from Weda Bay (Indonesia). Plant Soil 436, 543-563. https://doi.org/10.1007/s11104-019-03954-W

Luo, J., Yin, D., Cheng, H., Davison, W., Zhang, H., 2018. Plant Induced Changes to Rhizosphere Characteristics Affecting Supply of Cd to Noccaea caerulescens and Ni to Thlaspi goesingense. Environ. Sci. Technol. 52, 5085-5093. https://doi.org/10.1021/acs.est.7b04844

Ma, Y., Prasad, M.N.V., Rajkumar, M., Freitas, H., 2011. Plant growth promoting rhizobacteria and endophytes accelerate phytoremediation of metalliferous soils. Biotechnol. Adv. 29, 248-258. https://doi.org/10.1016/j.biotechadv.2010.12.001

Maciejewska, M., Całusińska, M., Cornet, L., Adam, D., Pessi, I., Malchair, S., Delfosse, P., Baurain, D., Barton, H., Carnol, M., Rigali, S., 2018. High-Throughput Sequencing Analysis of the Actinobacterial Spatial Diversity in Moonmilk Deposits. Antibiotics 7, 27. https://doi.org/10.3390/antibiotics7020027

Meerts, P., Grommesch, C., 2001. Soil seed banks in a heavy-metal polluted grassland at Prayon (Belgium). Plant Ecol. 11.

Merlot, S., de la Torre, V.S.G., Hanikenne, M., 2018. Physiology and Molecular Biology of Trace Element Hyperaccumulation.

Muehe, E.M., Weigold, P., Adaktylou, I.J., Planer-Friedrich, B., Kraemer, U., Kappler, A., Behrens, S., 2015. Rhizosphere microbial community composition affects cadmium and zinc uptake by the metal-hyperaccumulating plant Arabidopsis halleri. Appl. Environ. Microbiol. 81, $2173-2181$.

Page $11 / 18$ 
Mukhtar, S., Mirza, B.S., Mehnaz, S., Mirza, M.S., Mclean, J., Malik, K.A., 2018. Impact of soil salinity on the microbial structure of halophyte rhizosphere microbiome. World J. Microbiol. Biotechnol. 34. https://doi.org/10.1007/s11274-018-2509-5

Nouet, C., Charlier, J.-B., Carnol, M., Bosman, B., Farnir, F., Motte, P., Hanikenne, M., 2015. Functional analysis of the three HMA4 copies of the metal hyperaccumulator Arabidopsis halleri. J. Exp. Bot. 66, 5783-5795. https://doi.org/10.1093/jxb/erv280

Oksanen, J., Blanchet, F.G., Friendly, M., Kindt, R., Legendre, P., McGlinn, D., Minchin, P.R., O’Hara, R.B., Simpson, G.L., Solymos, P., Stevens, M.H.H., Szoecs, E., Wagner, H., 2018. vegan: Community Ecology Package.

Pauwels, M., Vekemans, X., Godé, C., Frérot, H., Castric, V., Saumitou-Laprade, P., 2012. Nuclear and chloroplast DNA phylogeography reveals vicariance among European populations of the model species for the study of metal tolerance, Arabidopsis halleri (Brassicaceae). New Phytol. 193, 916-928.

https://doi.org/10.1111/j.1469-8137.2011.04003.x

Praeg, N., Pauli, H., Illmer, P., 2019. Microbial Diversity in Bulk and Rhizosphere Soil of Ranunculus glacialis Along a High-Alpine Altitudinal Gradient. Front. Microbiol. 10, 1429. https://doi.org/10.3389/fmicb.2019.01429

Pruesse, E., 2012. SINA: high throughput multiple sequence alignment 15.

Pruesse, E., Quast, C., Knittel, K., Fuchs, B.M., Ludwig, W., Peplies, J., Glockner, F.O., 2007. SILVA: a comprehensive online resource for quality checked and aligned ribosomal RNA sequence data compatible with ARB. Nucleic Acids Res. 35, 7188-7196. https://doi.org/10.1093/nar/gkm864

Ramaut, J.L., 1964. Un aspect de la pollution atmosphérique: l'action des poussières de zinc sur les sols et les végétaux dans la région de Prayon. Nat. Belg. $45,133-145$.

Rees, F., Sterckeman, T., Morel, J.L., 2019. Biochar-assisted phytoextraction of Cd and Zn by Noccaea caerulescens on a contaminated soil: A four-year lysimeter study. Sci. Total Environ. 135654. https://doi.org/10.1016/j.scitotenv.2019.135654

Rosenfeld, C.E., Chaney, R.L., Martínez, C.E., 2018. Soil geochemical factors regulate Cd accumulation by metal hyperaccumulating Noccaea caerulescens (J. Presl \& C. Presl) F.K. Mey in field-contaminated soils. Sci. Total Environ. 616-617, 279-287. https://doi.org/10.1016/j.scitotenv.2017.11.016

Salt, D.E., Smith, R.D., Raskin, I., 1998. Phytoremediation. Annu Rev Plant Physiol Plant Mol Biol 26.

Sánchez-Marañón, M., Miralles, I., Aguirre-Garrido, J.F., Anguita-Maeso, M., Millán, V., Ortega, R., García-Salcedo, J.A., Martínez-Abarca, F., Soriano, M., 2017. Changes in the soil bacterial community along a pedogenic gradient. Sci. Rep. 7, 14593. https://doi.org/10.1038/s41598-017-15133-x

Schneider, T., Persson, D.P., Husted, S., Schellenberg, M., Gehrig, P., Lee, Y., Martinoia, E., Schjoerring, J.K., Meyer, S., 2013. A proteomics approach to investigate the process of Zn hyperaccumulation in Noccaea caerulescens (J \& C. Presl) F.K. Meyer. Plant J. 73, 131-142. https://doi.org/10.1111/tpj.12022

Schvartzman, M.S., Corso, M., Fataftah, N., Scheepers, M., Nouet, C., Bosman, B., Carnol, M., Motte, P., Verbruggen, N., Hanikenne, M., 2018. Adaptation to high zinc depends on distinct mechanisms in metallicolous populations of Arabidopsis halleri. New Phytol. 218, 269-282. https://doi.org/10.1111/nph.14949

Sessitsch, A., Kuffner, M., Kidd, P., Vangronsveld, J., Wenzel, W.W., Fallmann, K., Puschenreiter, M., 2013. The role of plant-associated bacteria in the mobilization and phytoextraction of trace elements in contaminated soils. Soil Biol. Biochem. 60, 182-194. https://doi.org/10.1016/j.soilbio.2013.01.012

Shahzad, Z., Gosti, F., Frérot, H., Lacombe, E., Roosens, N., Saumitou-Laprade, P., Berthomieu, P., 2010. The Five AhMTP1 Zinc Transporters Undergo Different Evolutionary Fates towards Adaptive Evolution to Zinc Tolerance in Arabidopsis halleri. PLoS Genet. 6, e1000911.

https://doi.org/10.1371/journal.pgen.1000911

Song, L., Pan, Z., Dai, Y., Chen, L., Zhang, L., Liao, Q., Yu, X., Guo, H., Zhou, G., 2020. Characterization and comparison of the bacterial communities of rhizosphere and bulk soils from cadmium-polluted wheat fields. PeerJ 8, e10302. https://doi.org/10.7717/peerj.10302

Spielmann, J., Ahmadi, H., Scheepers, M., Weber, M., Nitsche, S., Carnol, M., Bosman, B., Kroymann, J., Motte, P., Clemens, S., Hanikenne, M., 2020. The two copies of the zinc and cadmium ZIP6 transporter of Arabidopsis halleri have distinct effects on cadmium tolerance. Plant Cell Environ. 43, $2143-2157$. https://doi.org/10.1111/pce.13806

Stamatakis, A., 2014. RAxML version 8: a tool for phylogenetic analysis and post-analysis of large phylogenies. Bioinformatics 30, 1312-1313. https://doi.org/10.1093/bioinformatics/btu033

Stein, R.J., Höreth, S., de Melo, J.R.F., Syllwasschy, L., Lee, G., Garbin, M.L., Clemens, S., Krämer, U., 2017. Relationships between soil and leaf mineral composition are element-specific, environment-dependent and geographically structured in the emerging model Arabidopsis halleri. New Phytol. 213, 12741286. https://doi.org/10.1111/nph.14219

Sun, X., Zhou, Y., Tan, Y., Wu, Z., Lu, P., Zhang, G., Yu, F., 2018. Restoration with pioneer plants changes soil properties and remodels the diversity and structure of bacterial communities in rhizosphere and bulk soil of copper mine tailings in Jiangxi Province, China. Environ. Sci. Pollut. Res. 25, $22106-22119$.

https://doi.org/10.1007/s11356-018-2244-3

Page $12 / 18$ 
Talke, I.N., 2006. Zinc-Dependent Global Transcriptional Control, Transcriptional Deregulation, and Higher Gene Copy Number for Genes in Metal Homeostasis of the Hyperaccumulator Arabidopsis halleri. PLANT Physiol. 142, 148-167. https://doi.org/10.1104/pp.105.076232

Thomas, A.M., Segata, N., 2019. Multiple levels of the unknown in microbiome research. BMC Biol. 17, 48. https://doi.org/10.1186/s12915-019-0667-z

Ueno, D., Milner, M.J., Yamaji, N., Yokosho, K., Koyama, E., Clemencia Zambrano, M., Kaskie, M., Ebbs, S., Kochian, L.V., Ma, J.F., 2011. Elevated expression of TcHMA3 plays a key role in the extreme Cd tolerance in a Cd-hyperaccumulating ecotype of Thlaspi caerulescens: Cd transporter of hyperaccumulator. Plant $\mathrm{J}$. 66, 852-862. https://doi.org/10.1111/j.1365-313X.2011.04548.x

Ullah, A., Heng, S., Munis, M.F.H., Fahad, S., Yang, X., 2015. Phytoremediation of heavy metals assisted by plant growth promoting (PGP) bacteria: A review. Environ. Exp. Bot. 117, 28-40. https://doi.org/10.1016/j.envexpbot.2015.05.001

van de Mortel, J.E., Almar Villanueva, L., Schat, H., Kwekkeboom, J., Coughlan, S., Moerland, P.D., Ver Loren van Themaat, E., Koornneef, M., Aarts, M.G.M., 2006. Large Expression Differences in Genes for Iron and Zinc Homeostasis, Stress Response, and Lignin Biosynthesis Distinguish Roots of Arabidopsis thaliana and the Related Metal Hyperaccumulator Thlaspi caerulescens. Plant Physiol. 142, 1127-1147. https://doi.org/10.1104/pp.106.082073

Walters, W., Hyde, E.R., Berg-Lyons, D., Ackermann, G., Humphrey, G., Parada, A., Gilbert, J.A., Jansson, J.K., Caporaso, J.G., Fuhrman, J.A., Apprill, A., Knight, R., 2016. Improved Bacterial 16S rRNA Gene (V4 and V4-5) and Fungal Internal Transcribed Spacer Marker Gene Primers for Microbial Community Surveys. mSystems 1, e00009-15. https://doi.org/10.1128/mSystems.00009-15

Wang, B., Qiu, Y.-L., 2006. Phylogenetic distribution and evolution of mycorrhizas in land plants. Mycorrhiza 16, 299-363. https://doi.org/10.1007/s00572005-0033-6

Whiting, S.N., de Souza, M.P., Terry, N., 2001. Rhizosphere Bacteria Mobilize Zn for Hyperaccumulation by Thlaspi caerulescens. Environ. Sci. Technol. 35, 3144-3150. https://doi.org/10.1021/es001938v

Wickham, H., 2009. ggplot2: Elegant Graphics for Data Analysis. Springer-Verlag New York.

Wu, J., Zhao, F.-J., Ghandilyan, A., Logoteta, B., Guzman, M.O., Schat, H., Wang, X., Aarts, M.G.M., 2009. Identification and functional analysis of two ZIP metal transporters of the hyperaccumulator Thlaspi caerulescens. Plant Soil 325, 79-95. https://doi.org/10.1007/s11104-009-0151-6

Wu, L., Yang, B., Li, M., Chen, J., Xiao, Z., Wu, H., Tong, Q., Luo, X., Lin, W., 2019. Modification of Rhizosphere Bacterial Community Structure and Functional Potentials to Control Pseudostellaria heterophylla Replant Disease. Plant Dis. https://doi.org/10.1094/PDIS-04-19-0833-RE

Xiao, F., Yu, Y., Li, J., Juneau, P., Yan, Q., 2018. Necessary Sequencing Depth and Clustering Method to Obtain Relatively Stable Diversity Patterns in Studying Fish Gut Microbiota. Curr. Microbiol. 75, 1240-1246. https://doi.org/10.1007/s00284-018-1516-y

Yang, Y., Jiang, R.-F., Wang, W., Li, H.-F., 2011. Application of Rhizosphere Interaction of Hyperaccumulator Noccaea Caerulescens to Remediate CadmiumContaminated Agricultural Soil. Int. J. Phytoremediation 13, 933-945. https://doi.org/10.1080/15226514.2010.532181

Yu, K., Pieterse, C.M.J., Bakker, P.A.H.M., Berendsen, R.L., 2019. Beneficial microbes going underground of root immunity. Plant Cell Environ. pce.13632. https://doi.org/10.1111/pce.13632

Yue, Y., Shao, T., Long, X., He, T., Gao, X., Zhou, Z., Liu, Z., Rengel, Z., 2020. Microbiome structure and function in rhizosphere of Jerusalem artichoke grown in saline land. Sci. Total Environ. 724, 138259. https://doi.org/10.1016/j.scitotenv.2020.138259

Zhao, X., Sun, Y., Huang, J., Wang, H., Tang, D., 2020. Effects of soil heavy metal pollution on microbial activities and community diversity in different land use types in mining areas. Environ. Sci. Pollut. Res. 27, 20215-20226. https://doi.org/10.1007/s11356-020-08538-1

\section{Figures}




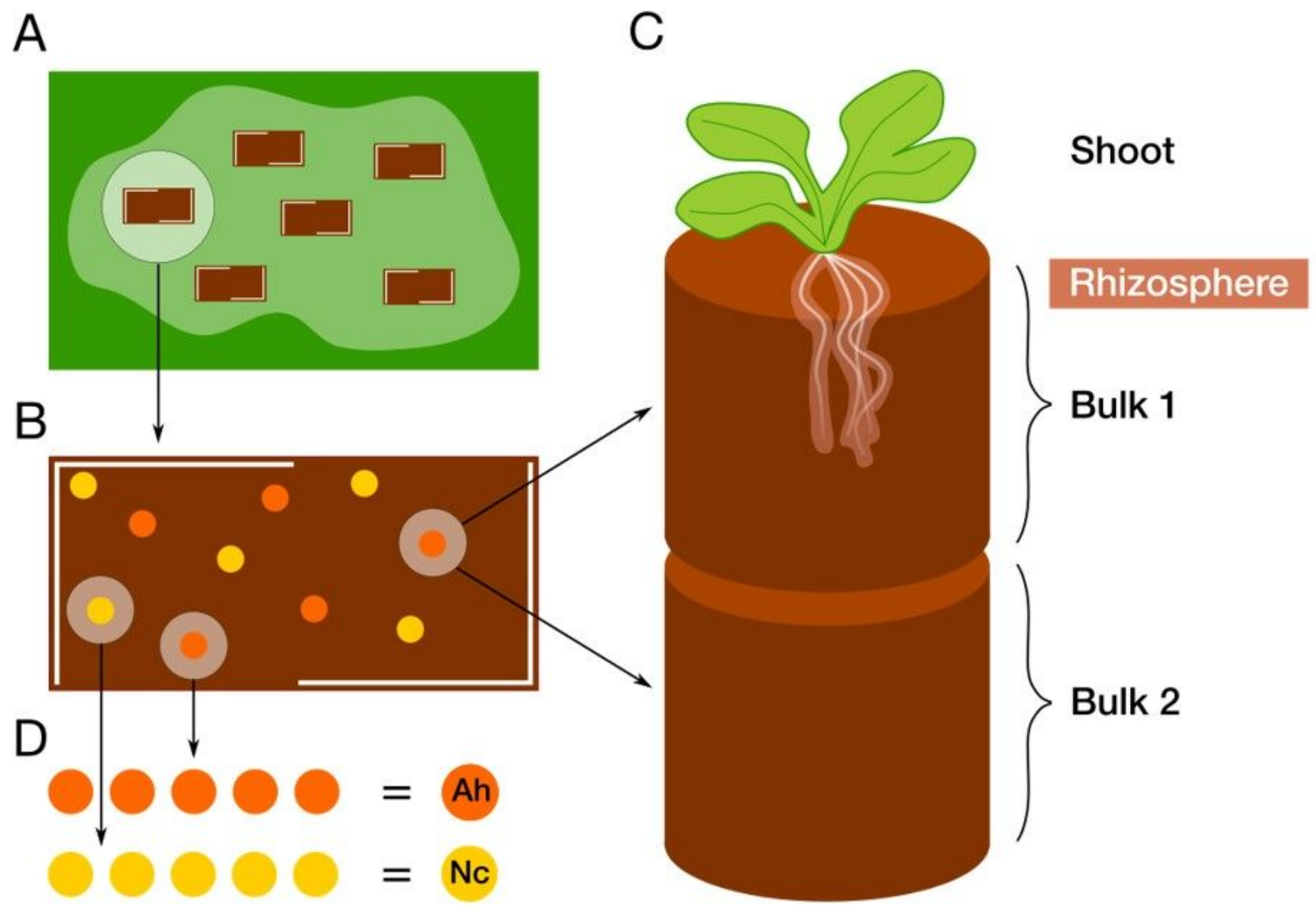

\section{Figure 1}

Sampling strategy. (A) 6 sampling plots (rectangles) were chosen on the site of Prayon. (B) Each plot consisted of a surface of $1 \mathrm{~m} \times 2 \mathrm{~m}$, in which five plants+cores were collected per species (Ah: A. halleri, Nc: N. caerulescens). (C) Each core was subdivided into four parts: the shoot, the rhizosphere, the upper part (bulk 1) and the bottom part (bulk 2). (D) Each part was pooled to constitute one sample per species and per plot. In total, $6 \times 3$ soil samples and 6 shoot samples were thus collected for each species. 

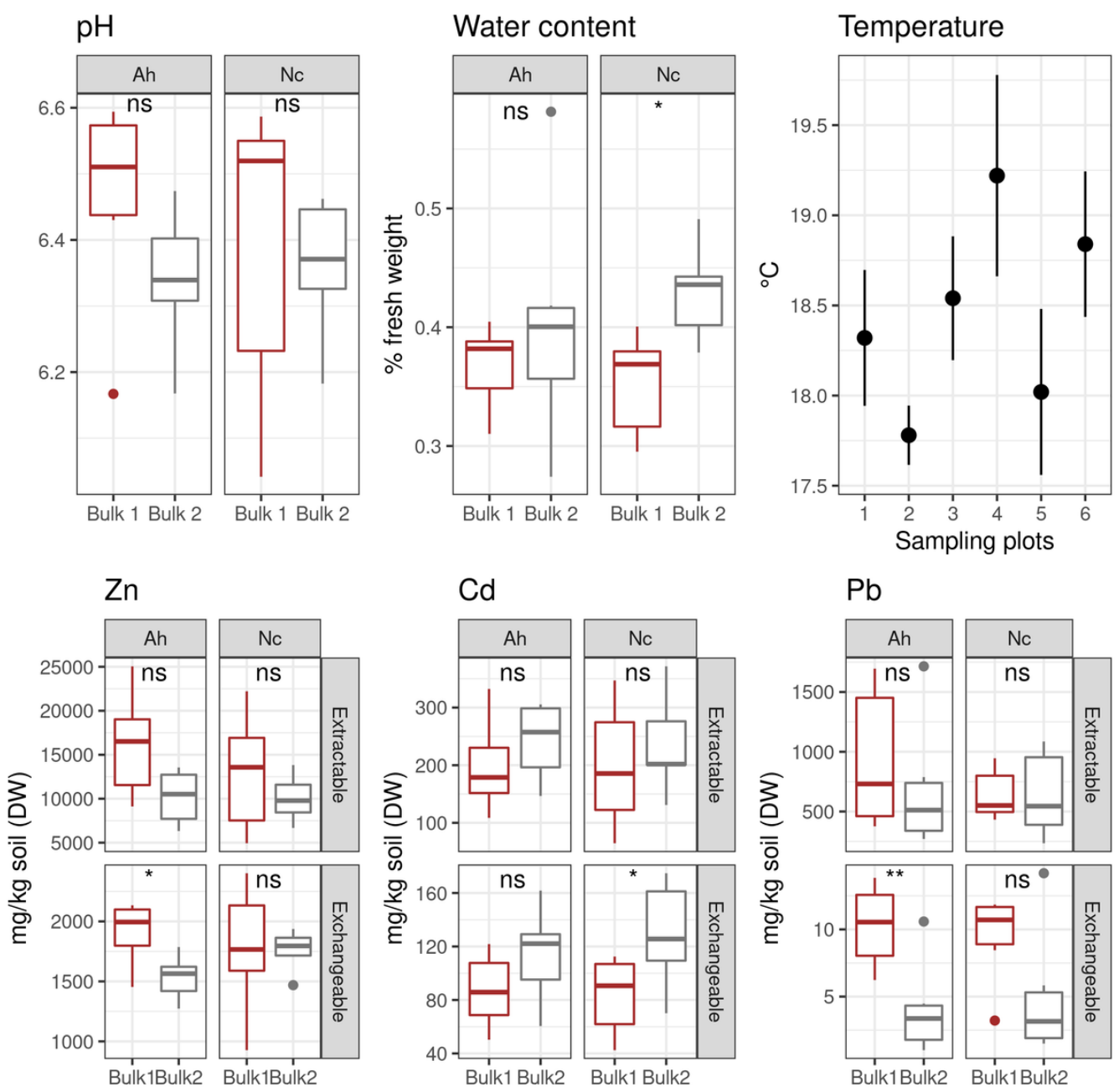

\section{Figure 2}

Selection of soil parameters for A. halleri (Ah) and N. caerulescens ( $\mathrm{Nc}$ ) samples (see Fig. S4 for the full series of figure panels). Extractable (HCl) and exchangeable $(\mathrm{BaCl} 2)$ element concentrations [mg kg-1 (DW)] in bulk samples are presented. Element concentration and water content values were measured from each soil sample. $\mathrm{pH}$ values are the means of five measures per sample. Temperature $\left({ }^{\circ} \mathrm{C}\right)$ values are the means of five measures and bars are standard deviations. Numbers 1 to 6 represent the six sampling plots. ns: $p>0.05, *$ : $<<=0.05, * *$ : $p<=0.01, * \star *$ : $p<=0.001, \star \star \star \star$ : $p<=0.0001$, from ANOVA tests. Boxes represent the first and third quartiles with the median in-between. Whiskers extends up to value $1.5 *$ interquartile range. Points are outliers. 

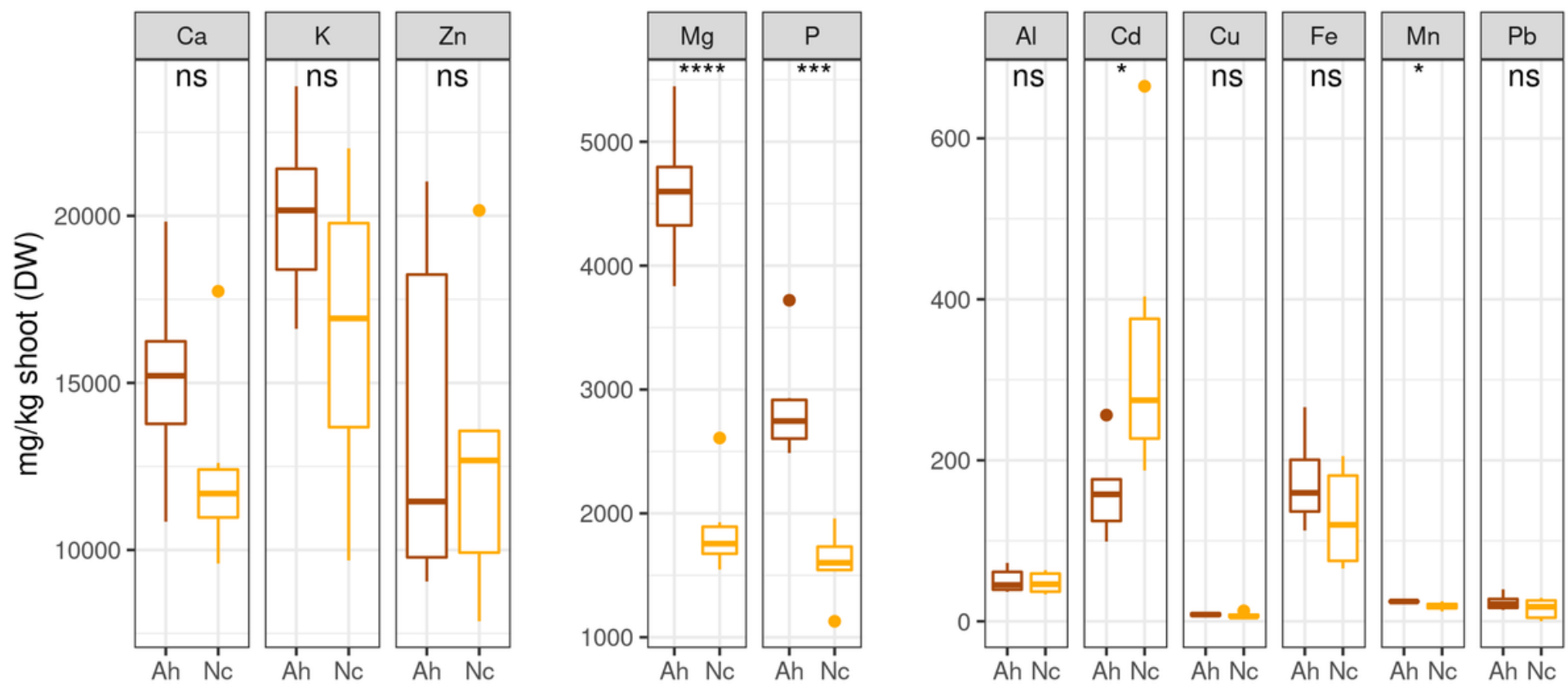

Figure 3

Element concentrations in the shoot of plants collected in Prayon (Belgium). Values are from six sampling plots, each including a pool of five plants, and are (a) above 6,000 mg kg-1 of shoot dry weight (DW), (b) between 1,000 and 6,000 mg kg-1 (DW) and (c) below 1,000 mg kg-1 (DW). Ah: A. halleri, Nc: N. caerulescens. Whiskers and other statistics details are as in Fig. 2.

A

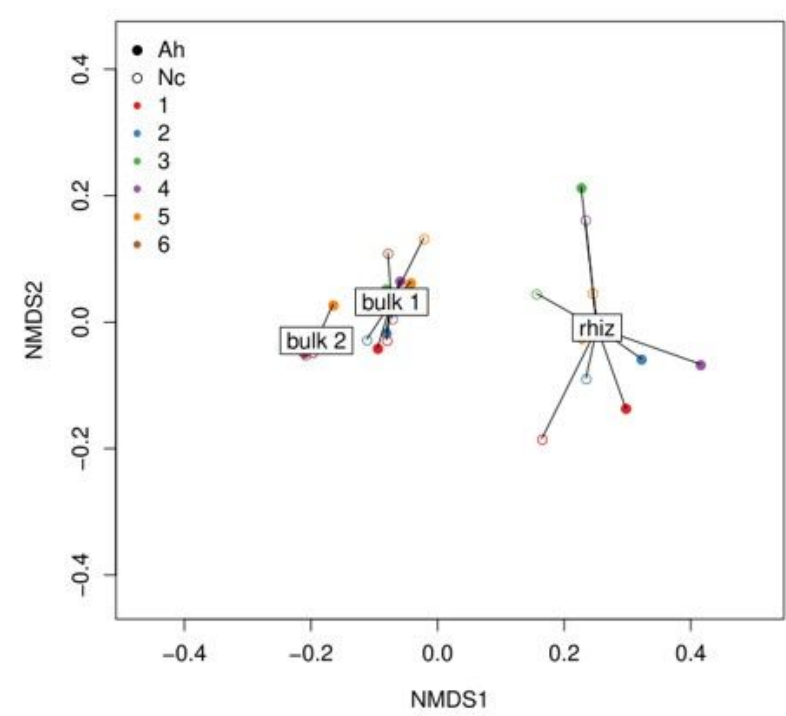

B Taxonomic diversity between rhizosphere samples

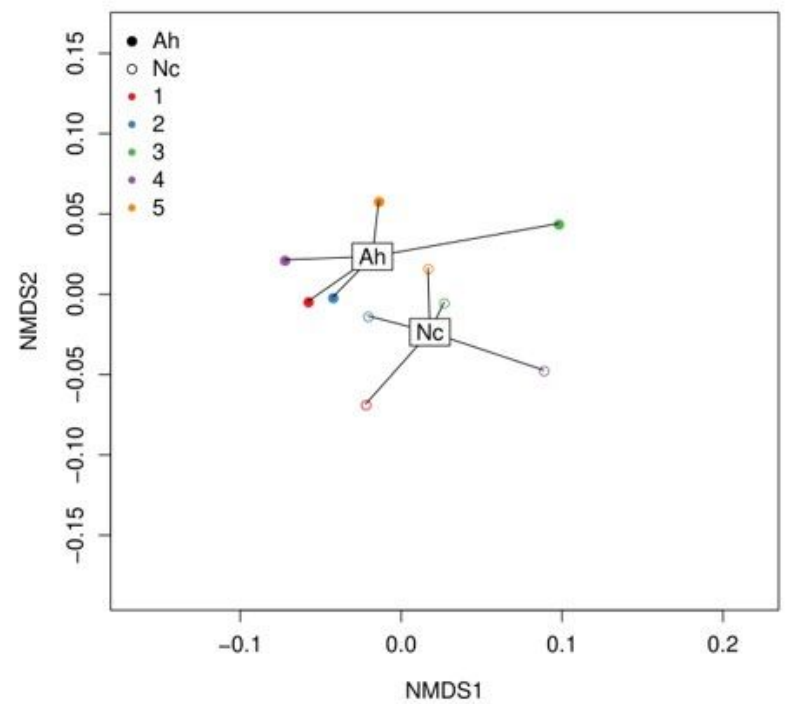

Figure 4

The NMDS analysis, representing the taxonomic diversity, was performed on the relative abundances of OTUs identified in each of the 30 samples (a) or in each of the 10 rhizosphere samples (b). Sample labels (rhiz, bulk 1 and bulk 2; Ah: A. halleri, Nc: N. caerulescens) are shown at the centroids of their respective projection. 1-6: sampling plots. 
A

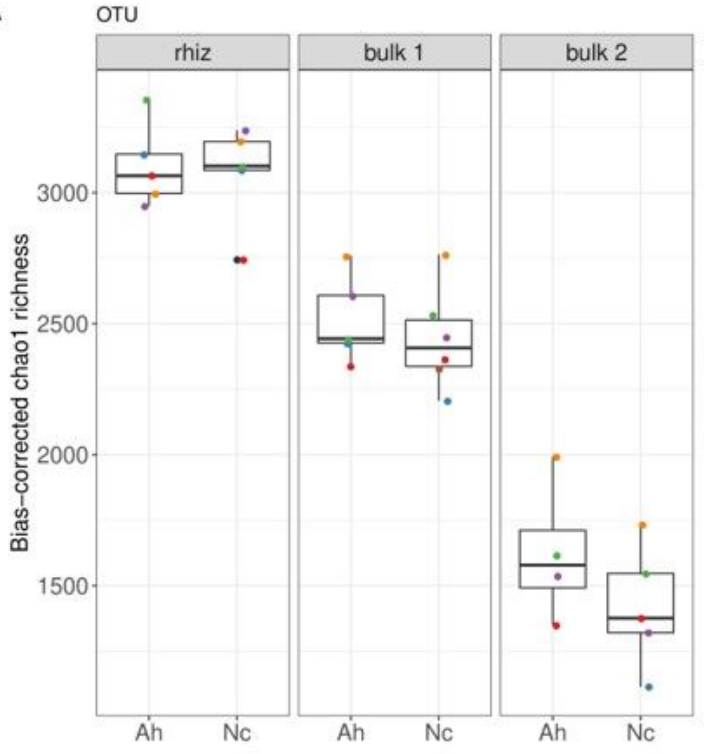

B

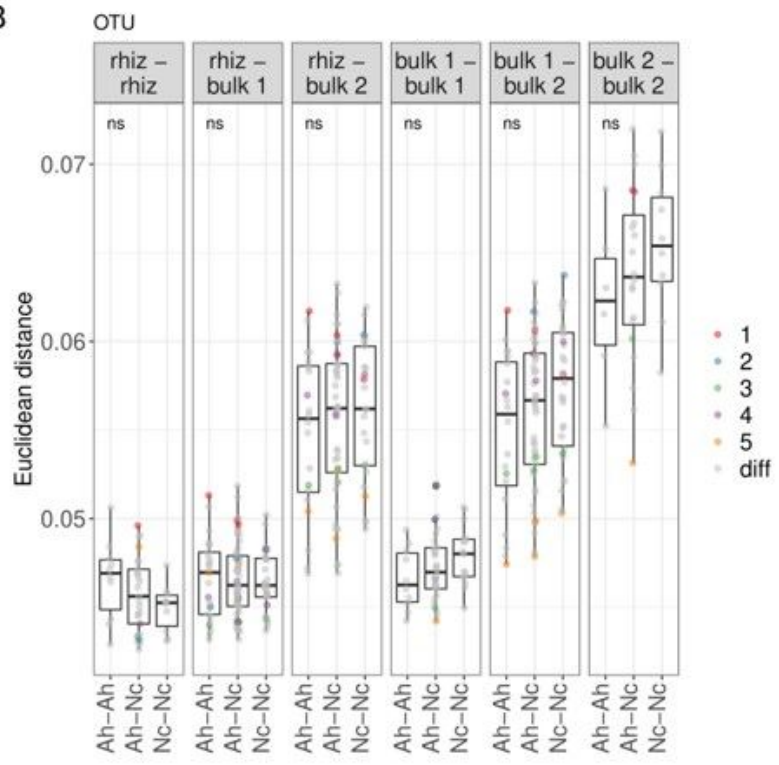

Figure 5

Distribution of alpha- and beta-diversity measures computed on OTUs. (a) Bias-corrected chao1 richness (alpha-diversity) are grouped by sample type (rhiz: rhizosphere, bulk 1 and bulk 2) and by plant species (Ah: A. halleri, Nc: N. caerulescens). (b) Euclidean distances (beta-diversity) are grouped by sample type comparison and by plant comparison. It takes into account the abundance of each OTU. Colors represent sampling plots (1-6). Grey (diff) represents comparison of samples from different sampling plots. Whiskers and other statistics details are as in Fig. 2.
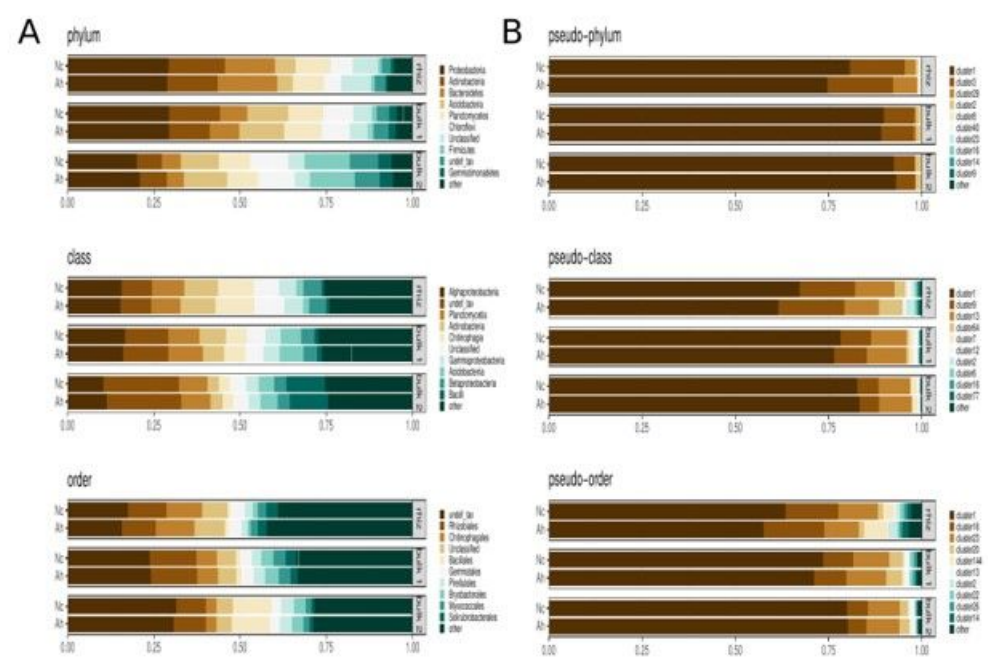

pse.do-dass
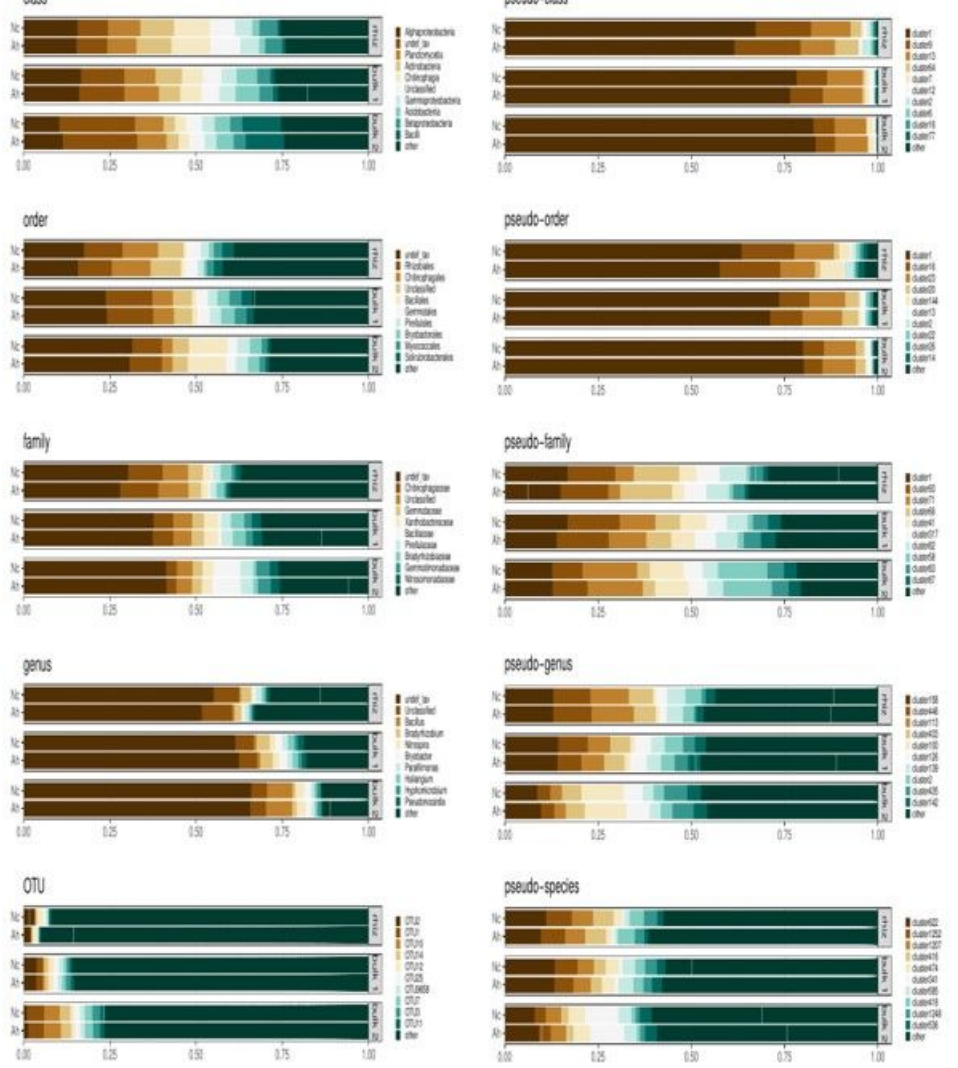
Composition of microbial communities highlighting the 10 most abundant taxa and pseudo-taxa at each (pseudo-)taxonomic level. The remaining taxa being lumped together as "others". (a) Taxonomy obtained with SILVA. "undef_tax" represents OTUs defined with SILVA but only at a higher taxonomic level. "Unclassified" represents OTUs always undefined at any taxonomic level. (b) Pseudo-taxonomy obtained by computing and segmenting a phylogenetic tree. Cluster numbers (i.e., pseudo-taxa) are purposely specific to each pseudo-level, as each clustering was computed independently. Ah: A. halleri, Nc: N. caerulescens.

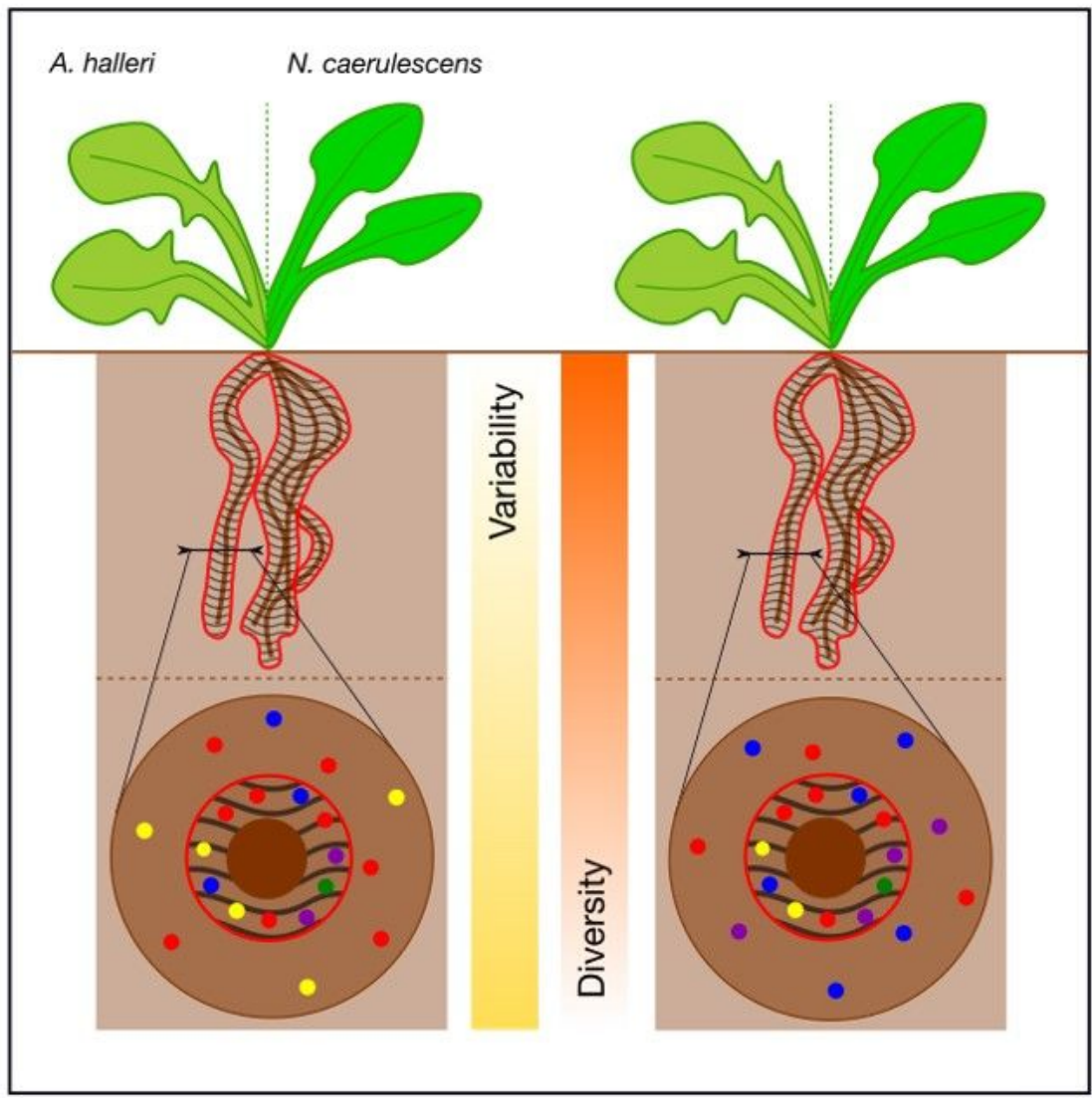

\section{Figure 7}

Diagram summarizing the microbial variability and diversity found in the rhizosphere (striped zone) and in bulks (in brown) with a slice (circle) containing different organisms (colored dots) in both A. halleri and N. caerulescens. Yellow and orange color shades represent variability and diversity gradients, respectively.

\section{Supplementary Files}

This is a list of supplementary files associated with this preprint. Click to download.

- PrayonSuppenvironmentalmicrobiome.pdf 